\title{
Iterated Hyperbolic Mixed Problems
}

By

\author{
Reiko Sakamoto, née Arima*
}

\section{Introduction}

Mixed problems for hyperbolic equations have been investigated by many authors, but mostly in the case when the equations are of second order or the case of one space dimension. In 1962, S. Agmon [1] established a priori estimates for solutions of general mixed problems of higher order hyperbolic equations with constant coefficients in a half space. In the case of variable coefficients, there are recent works of S. Mizohata [2] and of S. Miyatake [3]. In this paper, we study the conditions for solvability of higher order mixed problems by means of iteration procedure. We confined ourselves here to the case of half space, but it is not difficult to see that our method is also applicable to general bounded or unbounded domains.

In $\S 1$, we summarize $L^{2}$-energy method in elliptic general boundary value problems, due to Schechter [4]. We also clarify the dependence on the parameters in order to apply his results to unbounded domains. Here we consider even the case where boundary operators are not normal. The existence theorem is proved in appendix, using singular integral operators.

In $\S 2$, we shall study the iterated hyperbolic mixed problems on the basis of energy inequalities.

Received November 5, 1969.

Communicated by S. Matsuura.

* Department of Mathematics, Nara Women's University. 


\section{§1. Preliminary (Energy of Elliptic Type)}

\section{Fundamental energy inequalities.}

In this section we consider polynomials of one variable:

$$
\begin{array}{ll}
P(\xi)=a_{0} \xi^{m}+a_{1} \xi^{m-1}+\cdots+a_{m}=a_{0} \prod_{j=1}^{m}\left(\xi-\xi_{j}\right) & \left(a_{0} \neq 0\right), \\
P^{\prime}(\xi)=a_{0}^{\prime} \xi^{m}+a_{1}^{\prime} \xi^{m-1}+\cdots+a_{m}^{\prime}=a_{j=1}^{\prime n-1}\left(\xi-\xi_{j}^{\prime}\right) & \left(a_{0}^{\prime}=\cdots=a_{l-1}^{\prime}=0, a_{l}^{\prime} \neq 0\right) .
\end{array}
$$

We denote

$$
\dot{u}(\xi)=\int_{0}^{\infty} e^{-i x \xi} u(x) d x, \quad \omega_{k}=D^{k} u(0) \text { for } u(x) \in \mathscr{D}\left(R^{1}\right),
$$

where $D=\frac{1}{i} \frac{d}{d x}$, then we have

$$
\widehat{D^{k} u}(\xi)=\xi^{k} \bar{u}(\xi)+i\left(\omega_{k-1}+\xi \cdot \omega_{k-2}+\cdots+\xi^{k-1} \omega_{0}\right) .
$$

Let us denote

$$
\begin{gathered}
\widehat{P}(\xi, s)=a_{0} s^{m-1}+\left(a_{0} \xi+a_{1}\right) s^{m-2}+\cdots+\left(a_{0} \xi^{m-1}+\cdots+a_{m-1}\right), \\
\widehat{P}(\xi, \omega)=P\left(\xi, \omega_{0}, \cdots, \omega_{m-1}\right)=a_{0} \omega_{m-1}+\left(a_{0} \xi+a_{1}\right) \omega_{m-2}+\cdots \\
\cdots+\left(a_{0} \xi^{m-1}+\cdots+a_{m-1}\right) \omega_{0},
\end{gathered}
$$

then we have

$$
\widehat{P(D) u}(\xi)=P(\xi) u(\xi)+i \widehat{P}(\xi, \omega) .
$$

Now we denote

$$
\begin{aligned}
& \mathscr{P}(\xi, s)=\mathscr{Q}_{P P^{\prime}}(\xi, s)=P(\xi) \widehat{P}^{\prime}(\xi, s)-P^{\prime}(\xi) \widehat{P}(\xi, s), \\
& R=R_{P P^{\prime}}=a_{0}^{m-l} a_{l}^{\prime m} \prod_{i=1}^{m} \prod_{j=1}^{m-l}\left(\xi_{i}-\xi_{j}^{\prime}\right),
\end{aligned}
$$

then we have

Lemma 1.1. $\mathscr{P}$ is represented by

$$
\mathcal{L}(\xi, s)=\sum_{i, j=1}^{m} q_{i j} \xi^{\xi^{-i}} s^{m-j}=\left(\xi^{n-1}, \cdots, 1\right) Q\left(\begin{array}{c}
s^{m-1} \\
\vdots \\
1
\end{array}\right),
$$

where

$$
\begin{aligned}
& q_{i j}=\sum_{\substack{h+t=i+j-1 \\
h \geqslant \max (i, j) \\
t \min (i, j)-1}} a_{h} a_{t}^{\prime}-a_{h}^{\prime} a_{t}, \\
& \operatorname{det} Q=(-1)^{\frac{1}{2} m(m+1)} a_{0}^{l} R .
\end{aligned}
$$


Proof. From the definition of $\mathcal{Q}$, we have

$$
\begin{gathered}
\mathscr{Q}(\xi, s)=\left(a_{0} \xi^{m}+a_{1} \xi^{m-1}+\cdots+a_{m}\right)\left\{a_{0}^{\prime} s^{m-1}+\left(a_{0}^{\prime} \xi+a_{1}^{\prime}\right) s^{m-2}+\cdots\right. \\
\left.\cdots+\left(a_{0}^{\prime} \xi^{m-1}+\cdots+a_{m-1}^{\prime}\right)\right\} \\
-\left(a_{0}^{\prime} \xi^{m}+a_{1}^{\prime} \xi^{m-1}+\cdots+a_{m}^{\prime}\right)\left\{a_{0} s^{m-1}+\left(a_{0} \xi+a_{1}\right) s^{m-2}+\cdots\right. \\
\left.\cdots+\left(a_{0} \xi^{m-1}+\cdots+a_{m-1}\right)\right\} \\
=\left\{\left(a_{1} \xi^{m-1}+\cdots+a_{m}\right) a_{0}^{\prime}-\left(a_{1}^{\prime} \xi^{m-1}+\cdots+a_{m}^{\prime}\right) a_{0}\right\} s^{m-1} \\
\quad+\left\{\left(a_{2} \xi^{n-2}+\cdots+a_{m}\right)\left(a_{0}^{\prime} \xi+a_{1}^{\prime}\right)-\left(a_{2}^{\prime} \xi^{m-1}+\cdots+a_{m}^{\prime}\right)\left(a_{0} \xi+a_{1}\right)\right\} s^{m-2} \\
\quad+\cdots \cdots \cdots \cdots \cdots \cdots \cdots \\
\quad+\left\{a_{m}\left(a_{0}^{\prime} \xi^{m-1}+\cdots+a_{m-1}^{\prime}\right)-a_{m}^{\prime}\left(a_{0} \xi^{m-1}+\cdots+a_{m-1}\right)\right\} \\
=\sum_{\substack{h+t=i+j-1 \\
h \geqslant j>t}}\left(a_{h} a_{t}^{\prime}-a_{h}^{\prime} a_{t}\right) \xi^{m-i} s^{m-j} .
\end{gathered}
$$

Now suppose that $R=0$, that is, $\left\{P, P^{\prime}\right\}$ has a common root $\xi_{0}$, then we have $\mathscr{L}\left(\xi_{0}, s\right)=0$ identically, which means that $\operatorname{det} Q=0$. Remarking that $\operatorname{det} Q$ is a polynomial of degree $m(m-l)$ with respect to $\left(\xi_{1}, \cdots\right.$, $\left.\xi_{m}, \xi_{1}^{\prime}, \cdots, \xi_{m-l}^{\prime}\right)$, we have

$$
\operatorname{det} Q=c R \text {, }
$$

where $c$ is independent of $\left(\xi_{1}, \cdots, \xi_{m}, \xi_{1}^{\prime}, \cdots, \xi_{m-1}^{\prime}\right)$. It is shown easily that $c=(-1)^{\frac{1}{2} m(m+1)} a_{0}^{l}$.

For the present we consider

$$
\begin{aligned}
& P(\xi)=\xi^{m}+a_{1} \xi^{m-1}+\cdots+a_{m}, \\
& P^{\prime}(\xi)=\xi^{m}+a_{1}^{\prime} \xi^{m-1}+\cdots+a_{m}^{\prime},
\end{aligned}
$$

where we assume that

$$
\left|a_{j}\right|,\left|a_{j}^{\prime}\right| \leqslant C r^{j} \quad j=1,2, \cdots, m(C>0, r>0) .
$$

Lemma 1.2. We assume that

$$
\left|R_{P P^{\prime}}\right| \geqslant c r^{m^{2}}
$$

then we have

$$
|P(\xi)|+\left|P^{\prime}(\xi)\right| \geqslant c^{\prime}(|\xi|+r)^{m} \quad \text { for } \xi \in C^{1},
$$

where $c^{\prime}=c^{\prime}(C, c)$. The converse is also holds. 
Proof. Assume that

$$
\left|R_{P P^{\prime}}\right| \geqslant c r^{m^{2}}
$$

then we have

$$
\begin{gathered}
\left|\xi_{i}-\xi_{j}^{\prime}\right| \geqslant c_{0}(C, c) r \quad i, j=1,2, \cdots, m, \\
|P(\xi)|+\left|P^{\prime}(\xi)\right|=\prod_{j=1}^{m}\left|\xi-\xi_{j}\right|+\prod_{j=1}^{m}\left|\xi-\xi_{j}^{\prime}\right| \geqslant\left(\frac{1}{2} c_{0} r\right)^{m} \quad \text { for } \xi \in C^{1} .
\end{gathered}
$$

On the other hand, we have

$$
|P(\xi)|+\left|P^{\prime}(\xi)\right| \geqslant C_{0}(C)(|\xi|+r)^{m} \quad \text { for }|\xi| \geqslant C_{1}(C) r .
$$

Conversely assume that

$$
|P(\xi)|+\left|P^{\prime}(\xi)\right| \geqslant c^{\prime}(|\xi|+r)^{m} \quad \text { for } \xi \in C^{1} .
$$

Let $\xi=\xi_{j}$, then we have

$$
\left|P^{\prime}\left(\xi_{j}\right)\right| \geqslant c^{\prime}\left(\left|\xi_{j}\right|+r\right)^{m} \geqslant c^{\prime} r^{m}
$$

therefore

$$
|R|=\left|\prod_{j=1}^{m} P^{\prime}\left(\xi_{j}\right)\right| \geqslant c^{\prime m} \gamma^{m^{2}}
$$

Lemma 1. 3. We assume that

$$
|P(\xi)|+\left|P^{\prime}(\xi)\right| \geqslant c(|\xi|+r)^{m} \quad \text { for } \xi \in R^{1} \quad(c>0),
$$

then we have for $u \in \mathcal{E}_{L^{2}}^{m}(0, \infty)$

$$
\begin{aligned}
\frac{c^{2}}{2} \int_{0}^{\infty} & \left\{\left|D^{m} u(x)\right|^{2}+\cdots+r^{2 m}|u(x)|^{2}\right\} d x \\
\leqslant & \int_{0}^{\infty}\left\{|P(D) u(x)|^{2}+\left|P^{\prime}(D) u(x)\right|^{2}\right\} d x \\
& +C^{\prime}\left\{r\left|\omega_{m-1}\right|^{2}+\cdots+r^{2 m-1}\left|\omega_{0}\right|^{2}\right\},
\end{aligned}
$$

where $C^{\prime}=C^{\prime}(C, c)$.

Proof. Let

$$
P_{k}(\xi)=\frac{c}{\sqrt{2}} r^{k} \xi^{m-k}
$$

then

$$
|P(\xi)|^{2}+\left|P^{\prime}(\xi)\right|^{2}-\sum_{k-0}^{m}\left|P_{k}(\xi)\right|^{2} \geqslant \frac{c^{2}}{2}(|\xi|+r)^{2 m} .
$$


Now we have

$$
\begin{aligned}
& |\widehat{P(D) u}(\xi)|^{2}+\left|\widehat{P^{\prime}(D) u(\xi)}\right|^{2}-\sum_{k=0}^{m} \mid \widehat{P}_{k}\left(\left.\widehat{D) u(\xi)}\right|^{2}\right. \\
& =|P(\xi) u(\xi)+i \widehat{P}(\xi, \omega)|^{2}+\left|P^{\prime}(\xi) \grave{u}(\xi)+i \widehat{P^{\prime}}(\xi, \omega)\right|^{2} \\
& -\sum_{k=0}^{m}\left|P_{k}(\xi) \bar{u}(\xi)+i \widehat{P}_{k}(\xi, \omega)\right|^{2} \\
& =\left(|P|^{2}+\left|P^{\prime}\right|^{2}-\sum_{k=0}^{m}\left|P_{k}\right|^{2}\right)|\bar{u}|^{2}+2 \operatorname{Re}\left\{i\left(\widehat{P} \bar{P}+\widehat{P}^{\prime} \bar{P}^{\prime}-\sum_{k-0}^{m} \widehat{P}_{k} \bar{P}_{k}\right) \bar{u}\right\} \\
& +\left(|\widehat{P}|^{2}+\left|\widehat{P}^{\prime}\right|^{2}-\sum_{k=0}^{m}\left|\widehat{P}_{k}\right|^{2}\right) \\
& =\left(|P|^{2}+\left|P^{\prime}\right|^{2}-\sum_{k=0}^{m}\left|P_{k}\right|^{2}\right)\left|u+i \frac{\widehat{P} \bar{P}+\widehat{P}^{\prime} \bar{P}^{\prime}-\sum \widehat{P}_{k} \bar{P}_{k}}{|P|^{2}+\left|P^{\prime}\right|^{2}-\sum\left|P_{k}\right|^{2}}\right|^{2} \\
& +\frac{\left|\mathscr{Q}_{P F^{\prime}}\right|^{2}-\sum\left|\mathscr{Q}_{P P_{k}}\right|^{2}-\sum\left|\mathscr{Q}_{P^{\prime} P_{k}}\right|^{2}+\sum_{j, k}\left|\mathscr{Q}_{P_{P} P_{k}}\right|^{2}}{|P|^{2}+\left|P^{\prime}\right|^{2}-\sum\left|P_{k}\right|^{2}} .
\end{aligned}
$$

From the representation of $\mathscr{P}$ in Lemma 1.1 ,

$$
\begin{gathered}
\left.\left.\int_{-\infty}^{\infty}\left\{|\widehat{P(D) u}(\xi)|^{2}+\left|\widehat{P^{\prime}(D) u(\xi)}\right|^{2}-\sum_{k=0}^{m} \mid \widehat{P_{k}(D)}\right) \overline{u(\xi)}\right|^{2}\right\} d \xi \\
\geqslant-C^{\prime}(C, c) \int_{-\infty}^{\infty} \frac{\left(\sum_{i, j=1}^{m}|\xi|^{m^{-2}} r^{i \vdash j-1}\left|\omega_{m-j}\right|\right)^{2}}{(|\xi|+r)^{2 m}} d \xi \\
\geqslant-C^{\prime \prime}(C, c) \sum_{j=1}^{m}\left(r^{j-\frac{1}{2}}\left|\omega_{m-j}\right|\right)^{2} .
\end{gathered}
$$

\section{Lemma 1.4. We assume that}

$$
\left|R_{P P^{\prime}}\right| \geqslant c r^{m^{2}}
$$

then we have for $u \in \mathcal{E}_{L^{2}}{ }^{2}(0, \infty)$

$$
c^{\prime}\left\{r\left|\omega_{m-1}\right|^{2}+\cdots+r^{2 m-1}\left|\omega_{0}\right|^{2}\right\} \leqslant \int_{0}^{\infty}\left\{|P(D) u(x)|^{2}+\left|P^{\prime}(D) u(x)\right|^{2}\right\} d x,
$$

where $c^{\prime}=c^{\prime}(C, c)$.

Proof. In the same way as in Lemma 1.3, we have

$$
\begin{aligned}
& |\overline{P(D) u}(\xi)|^{2}+\left|\overline{P^{\prime}(D) u(\xi)}\right|^{2} \\
& =\left(|P|^{2}+\left|P^{\prime}\right|^{2}\right)\left|u+i \frac{\widehat{P} \bar{P}+\widehat{P}^{\prime} \bar{P}^{\prime}}{|P|^{2}+\left|P^{\prime}\right|^{2}}\right|^{2}+\frac{\left|\mathscr{P}_{P P^{\prime}}\right|^{2}}{|P|^{2}+\left|P^{\prime}\right|^{2}} \text {. }
\end{aligned}
$$

From Lemma 1.2, we have

$$
|P|^{2}+\left|P^{\prime}\right|^{2} \geqslant c^{\prime}(C, c)(|\xi|+r)^{2 m} \quad \text { for } \xi \in R^{1} .
$$


Hence we denote

where

$$
|P|^{2}+\left|P^{\prime}\right|^{2}=\prod_{j=1}^{m}\left(\xi-\xi_{j}^{\prime \prime}\right) \cdot 2 \prod_{j=m+1}^{2 m}\left(\xi-\xi_{j}^{\prime \prime}\right)=P_{+}^{\prime \prime} P_{-}^{\prime \prime},
$$

$$
\operatorname{Im} \xi_{j}^{\prime \prime}>c_{0}(C, c) r>0 \quad j=1,2, \cdots, m .
$$

Then we have

$$
\begin{aligned}
\int_{-\infty}^{\infty} & \frac{|\mathscr{L}(\xi, \omega)|^{2}}{|P(\xi)|^{2}+\left|P^{\prime}(\xi)\right|^{2}} d \xi=\left(\bar{\omega}_{m-1}, \cdots, \bar{\omega}_{0}\right) Q^{*} \\
& \times\left(\int_{-\infty}^{\infty} \frac{\xi^{n^{-\prime}-j}}{|P(\xi)|^{2}+\left|P^{\prime}(\xi)\right|^{2}} d \xi\right)_{i, j=1, \cdots, m} Q\left(\begin{array}{c}
\omega_{m-1} \\
\vdots \\
\omega_{0}
\end{array}\right) \\
= & \left(\bar{\omega}_{m-1}, \cdots, \bar{\omega}_{0}\right) Q^{*} E_{0} Q\left(\begin{array}{c}
\omega_{m-1} \\
\vdots \\
\omega_{0}
\end{array}\right),
\end{aligned}
$$

where we have

$$
\operatorname{det} E_{0}=\frac{(2 \pi i)^{m}}{\prod_{j=1}^{m} P_{-}^{\prime \prime}\left(\xi_{j}^{\prime \prime}\right)}
$$

Now we denote

$$
\Omega_{m-j}=r^{-\frac{1}{2}} \omega_{m-j} \quad j=1, \cdots, m
$$

and

$$
\left(\overline{\boldsymbol{\omega}}_{m-1}, \cdots, \bar{\omega}_{0}\right) Q^{*} E_{0} Q\left(\begin{array}{c}
\omega_{m-1} \\
\vdots \\
\omega_{0}
\end{array}\right)=\left(\bar{\Omega}_{m-1}, \cdots, \overline{\Omega_{0}}\right) E_{1}\left(\begin{array}{c}
\Omega_{m-1} \\
\vdots \\
\Omega_{0}
\end{array}\right)
$$

then we have, from Lemma 1.1, that the absolute value of each element of $E_{1}$ is bounded by $C^{\prime}(C, c), E_{1}$ is positive, and $\left|\operatorname{det} E_{1}\right|>c_{1}(C, c)>0$.

Lemma 1.5. We assume that $\operatorname{Im} \xi_{j}<0(j=1, \cdots, m)$ and

$$
|P(\xi)| \geqslant c(|\xi|+r)^{m} \quad \text { for } \xi \in R^{1} .
$$

Then we have, for $u \in \mathcal{E}_{L^{2}}^{m}(0, \infty)$,

$$
\begin{aligned}
& \int_{0}^{\infty}|P(D) u(x)|^{2} d x \geqslant c^{2} \int_{0}^{\infty}\left\{\left|D^{m} u(x)\right|^{2}\right. \\
& \left.\quad+r^{2}\left|D^{m-1} u(x)\right|^{2}+\cdots+r^{2 m}|u(x)|^{2}\right\} d x .
\end{aligned}
$$

Proof. The unique solution of $P(D) u(x)=f(x)(x>0)$ is given by 


$$
u(x)=\frac{1}{2 \pi} \int_{-\infty}^{\infty} e^{i x \xi} \frac{\widetilde{f}(\xi)}{P(\xi)} d \xi \quad(x>0),
$$

then we have

$$
r^{j} D^{n-j} u(x)=\frac{1}{2 \pi} \int_{-\infty}^{\infty} e^{i x \xi} \frac{r^{j} \xi^{n-j} \widetilde{f}(\xi)}{P(\xi)} d \xi \quad(x>0) .
$$

Therefore we have

$$
\int_{0}^{\infty} \sum_{j=0}^{m}\left|r^{j} D^{m-i} u(x)\right|^{2} d x=\frac{1}{2 \pi} \int_{-\infty}^{\infty} \sum_{j=0}^{n}\left|\frac{r^{\jmath} \xi^{m-\jmath} \widetilde{f}(\xi)}{P(\xi)}\right|^{2} d \xi \leqslant \frac{1}{c^{2}} \int_{0}^{\infty}|f(x)|^{2} d x .
$$

Here we introduce

$$
M_{K}(\xi)=\xi+i K r \quad(K>0),
$$

then we have from Lemma 1.5

$$
\begin{aligned}
c_{K} \int_{0}^{\infty} \sum_{j=0}^{m}\left|\gamma^{j} D^{s-j} u(x)\right|^{2} d x & \leqslant \int_{0}^{\infty}\left|M_{K}(D)^{s} u(x)\right|^{2} d x \\
& \leqslant c_{K}^{\prime} \int_{0}^{\infty} \sum_{j=0}^{m}\left|r^{j} D^{s-\jmath} u(x)\right|^{2} d x
\end{aligned}
$$

Finally we consider the general case when

$$
\begin{aligned}
& P(\xi)=\xi^{m}+a_{1} \xi^{m^{-1}}+\cdots+a_{m}=\prod_{j-1}^{m}\left(\xi-\xi_{j}\right), \\
& P^{\prime}(\xi)=\xi^{\prime n^{\prime}}+a_{1}^{\prime} \xi^{m^{\prime}-1}+\cdots+a_{m^{\prime}}^{\prime}=\prod_{j=1}^{m^{\prime}}\left(\xi-\xi_{j}^{\prime}\right),
\end{aligned}
$$

where we assume that

$$
\left|a_{j}\right| \leqslant C r^{j}(j=1, \cdots, m), \quad\left|a_{j}^{\prime}\right| \leqslant C r^{j}\left(j=1, \cdots, m^{\prime}\right) .
$$

Proposition 1.1. We assume that

$$
|P(\xi)|^{m^{\prime}}+\left|P^{\prime}(\xi)\right|^{m} \geqslant c(|\xi|+r)^{m m^{\prime}} \quad \text { for } \xi \in R^{1} \quad(c>0) .
$$

Then we have for $u \in \mathcal{E}_{L^{2}}^{s}(0, \infty)$

$$
\begin{aligned}
& \int_{0}^{\infty} \sum_{j=0}^{s}\left|r^{j} D^{s-j} u(x)\right|^{2} d x \leqslant C_{s} \int_{0}^{\infty}\left\{\sum_{j=0}^{s-m}\left|r^{j} D^{s-m-j} P(D) u(x)\right|^{2}\right. \\
& \left.\quad+\sum_{j=0}^{s-m \prime}\left|r^{j} D^{s-m^{\prime}-j} P^{\prime}(D) u(x)\right|^{2}\right\} d x+C_{s}\left\{r\left|\omega_{s-1}\right|^{2}+\cdots+r^{2 s-1}\left|\omega_{0}\right|^{2}\right\}
\end{aligned}
$$

where $s \geqslant \max \left(m, m^{\prime}\right)$ and $C_{s}=C_{s}(C, c)$. 
Proof. Let

$$
\begin{aligned}
& Q(\xi)=M_{1}(\xi)^{s-m} P(\xi), \\
& Q^{\prime}(\xi)=M_{1}(\xi)^{s-m^{\prime}} P^{\prime}(\xi),
\end{aligned}
$$

and apply Lemma 1.3 to $\left\{Q, Q^{\prime}\right\}$.

Proposition 1.2. We assume that $P, P^{\prime}$ are decomposed in such a way that

$$
P=P_{0} P_{(-)}, \quad P^{\prime}=P_{0}^{\prime} P_{(-)}^{\prime},
$$

where all the roots of $P_{(-)}$and $P_{(-)}^{\prime}$ have negative imaginary parts and

$$
\begin{aligned}
& \left|P_{(-)}(\xi)\right| \geqslant c(|\xi|+r)^{m-\mu},\left|P_{(-)}^{\prime}(\xi)\right| \geqslant c(|\xi|+r)^{m^{\prime}-\mu^{\prime}} \text { for } \xi \in R^{1}, \\
& \left|R_{P_{0} P_{0}^{\prime}}\right| \geqslant c r^{\mu \mu \prime} .
\end{aligned}
$$

Then we have if $u$ belongs to $\mathcal{E}_{L^{2}}^{s}(0, \infty)$,

$$
\begin{aligned}
& c_{s} \int_{0}^{\infty} \sum_{j=0}^{s}\left|r^{j} D^{s-j} u(x)\right|^{2} d x \\
& \quad \leqslant \int_{0}^{\infty}\left\{\sum_{j=0}^{s-n}\left|r^{j} D^{s-m-j} P(D) u(x)\right|^{2}+\sum_{j=0}^{s-m^{\prime}}\left|r^{j} D^{s-m^{\prime-j}} P^{\prime}(D) u(x)\right|^{2}\right\} d x,
\end{aligned}
$$

where $s \geqslant \max \left(m, m^{\prime}\right)$ and $c_{s}=c_{s}(C, c)$.

Proof. We have

$$
\left|\xi_{i}\right|,\left|\xi_{j}^{\prime}\right| \leqslant C_{0}(C) r \quad i=1, \cdots, m, \quad j=1, \cdots, m^{\prime} .
$$

We denote

$$
\begin{aligned}
& Q(\xi)=M_{2 C_{0}}(\xi)^{s-\mu} P_{0}(\xi), \\
& Q^{\prime}(\xi)=M_{3 C_{0}}(\xi)^{s-\mu} P_{0}^{\prime}(\xi),
\end{aligned}
$$

then

$$
\left|R_{Q Q^{\prime}}(\xi)\right| \geqslant\left(C_{0} \gamma\right)^{s-\mu \mu^{\prime}}\left|R_{P_{0} F_{0}^{\prime}}\right| \geqslant c C_{0}^{s 2-\mu \mu^{\prime}} \gamma^{s^{2}} .
$$

Therefore we have from Lemma 1.4

$$
\begin{aligned}
& c_{0}(C, c) \int_{0}^{\infty} \sum_{j=0}^{s}\left|r^{j} D^{s-j} u(x)\right|^{2} d x \\
& \quad \leqslant \int_{0}^{\infty}\left\{\sum_{j=0}^{s-\mu}\left|\gamma^{j} D^{s-\mu-j} P_{0}(D) u(x)\right|^{2}+\sum_{j=0}^{s-\mu^{\prime}}\left|\gamma^{j} D^{s-\mu^{\prime-j}} P_{0}^{\prime}(D) u(x)\right|^{2}\right\} d x .
\end{aligned}
$$

On the other hand, we have from Lemma 1.5 


$$
\begin{array}{rl}
\int_{0}^{\infty} \mid M_{1}(D)^{s-m} & \left.P(D) u(x)\right|^{2} d x=\int_{0}^{\infty}\left|M_{1}(D)^{s-m} P_{(-)}(D) P_{0}(D) u(x)\right|^{2} d x \\
& \geqslant c_{1}(C, c) \int_{0}^{\infty} \sum_{j=0}^{s-\mu}\left|r^{j} D^{s-\mu^{-j}} P_{0}(D) u(x)\right|^{2} d x \\
\int_{0}^{\infty} \mid M_{1}(D)^{s-m^{\prime}} & \left.P^{\prime}(D) u(x)\right|^{2} d x \\
& \geqslant c_{1}(C, c) \int_{0}^{\infty} \sum_{j=0}^{s-\mu^{\prime}}\left|r^{j} D^{s-l^{\prime}-j} P_{0}^{\prime}(D) u(x)\right|^{2} d x
\end{array}
$$

\section{Hyperbolic polynomials.}

Let $P\left(\tau, \xi, \eta_{1}, \eta_{2}, \cdots, \eta_{n-1}\right)$ be a homogeneous polynomial of degree $m$, where the coefficients of $\tau^{m}$ and $\xi^{m}$ are not zero, which we denote

$$
\begin{aligned}
P & (\tau, \xi, \eta)=\sum_{i+j+|\nu|=m} p_{i j \nu} \tau^{2} \xi^{j} \eta^{\nu} \\
& =p_{0} \tau^{m}+p_{1}(\xi, \eta) \tau^{m-1}+\cdots+p_{m}(\xi, \eta)=p_{0} \prod_{j=1}^{m}\left(\tau-\tau_{j}(\xi, \eta)\right) \quad\left(p_{0} \neq 0\right) \\
& =a_{0} \xi^{m}+a_{1}(\tau, \eta) \xi^{m-1}+\cdots+a_{m}(\tau, \eta)=a_{0} \prod_{j=1}^{m}\left(\xi-\xi_{j}(\tau, \eta)\right) \quad\left(a_{0} \neq 0\right) .
\end{aligned}
$$

We assume that $\left\{\tau_{j}(\xi, \eta)\right\}_{j=1, \cdots, m}$ are real for $(\xi, \eta) \in R^{n}$, which we say that $P(\tau, \xi, \eta)$ is hyperbolic with respect to $\tau$. Then $\mu$ of $\left\{\tau_{1}, \cdots, \tau_{m}\right\}$ are negative and the others positive for $\xi>0$ and $\eta=0$, therefore $\mu$ of $\left\{\xi_{1}, \cdots, \xi_{m}\right\}$ have positive imaginary parts and the others negative ones for $\operatorname{Im} \tau<0$ and $\eta \in R^{n-1}$, whicn we denote by $\left\{\xi_{1}^{+}, \cdots, \xi_{\mu}^{+}\right\}$and $\left\{\xi_{1}^{-}, \cdots\right.$, $\left.\xi_{m-\mu}^{-}\right\}$respectively. Here we denote

$$
P_{+}(\tau, \xi, \eta)=\prod_{j=1}^{\mu}\left(\xi-\xi_{j}^{+}(\tau, \eta)\right), \quad P_{-}(\tau, \xi, \eta)=a_{0} \prod_{j=1}^{m-\mu}\left(\xi-\xi_{j}^{-}(\tau, \eta)\right) .
$$

Next we consider another hyperbolic polynomial $P^{\prime}$ of homogeneous degree $m^{\prime}$ :

$$
\begin{aligned}
P^{\prime}(\tau, \xi, \eta) & =\sum_{i+j+|\nu|=m^{\prime}} p_{j_{\nu}}^{\prime} \tau^{i} \xi^{j} \eta^{\nu}=p_{0}^{\prime} \prod_{j=1}^{m^{\prime}}\left(\tau-\tau_{j}^{\prime}(\xi, \eta)\right) \\
& =a_{0}^{\prime} \prod_{j=1}^{m^{\prime}}\left(\xi-\xi_{j}^{\prime}(\tau, \eta)\right)=P_{+}^{\prime}(\tau, \xi, \eta) P_{-}^{\prime}(\tau, \xi, \eta) .
\end{aligned}
$$

We denote the resultant of $\left\{P, P^{\prime}\right\}$ with respect to $\tau$ by

$$
R_{P P}^{0},(\xi, \eta)=R^{0}(\xi, \eta)=p_{0}^{m \prime} p_{0}^{\prime m} \prod_{i=1}^{m} \prod_{j-1}^{m^{\prime}}\left(\tau_{\imath}(\xi, \eta)-\tau_{j}^{\prime}(\xi, \eta)\right),
$$

and that of $\left\{P_{+}, P_{+}^{\prime}\right\}$ with respect to $\xi$ by 


$$
R_{P_{+} P_{+}^{\prime}}(\tau, \eta)=R^{+}(\tau, \eta)=\prod_{i=1}^{\mu} \prod_{j=1}^{\mu \prime}\left(\xi_{i}^{+}(\tau, \eta)-\xi_{j}^{+}(\tau, \eta)\right) .
$$

Here we assume that

$$
\begin{aligned}
& \text { (I) } \quad \inf _{\substack{(\xi, \eta) \in R^{n} \\
|\xi|+|\eta|=1}}\left|R^{0}(\xi, \eta)\right|=k_{1} \neq 0, \\
& \text { (II) } \quad \inf _{\substack{\operatorname{Im} \tau<0, \eta n \in R^{n-1} \\
|\tau|+|\eta|=1}}\left|R^{+}(\tau, \eta)\right|=k_{2} \neq 0 \text {. }
\end{aligned}
$$

Now we denote

$$
\max \left(\left|p_{i j \nu}\right|,\left|p_{i j \nu}^{\prime}\right|,\left|p_{0}\right|^{-1},\left|p_{0}^{\prime}\right|^{-1},\left|a_{0}\right|^{-1},\left|a_{0}^{\prime}\right|^{-1}\right)=K
$$

then we have

Lemma 2.1. (I) is equivalent to the following: ihere exists $h>0$ such that

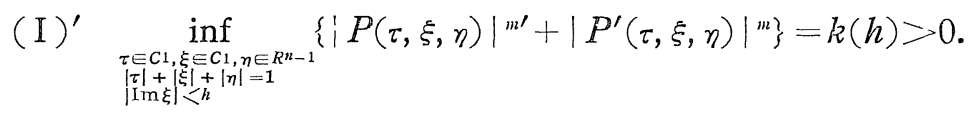

More precisely, if we assume (I), then there exists $h=h\left(K, k_{1}\right)>0$ and $k(h)<h$, and if we assume $(I)^{\prime}$, then $k_{1}>c^{\prime}(K, k(h))>0$.

Proof. Assume (I). Since $R_{P m^{\prime} P{ }^{\prime} m}^{0}=\left(R_{P I^{\prime}}^{0}\right)^{m n^{\prime}}$, we have from Lemma 1.2

$$
\inf _{\substack{\tau \in C 1,(\xi, \eta) \in R^{\prime \prime} \\|\tau|+|\xi|+|\eta|=1}}\left\{|P(\tau, \xi, \eta)|^{m^{\prime} \prime}+\left|P^{\prime}(\tau, \xi, \eta)\right|^{m}\right\} \geqslant c\left(K, k_{1}\right)>0 .
$$

Now we denote, for $\xi \in C^{1}, \operatorname{Re} \xi=\alpha, \operatorname{Im} \xi=\beta$, then we have

$$
\begin{aligned}
& P(\tau, \alpha+i \beta, \eta)=P(\tau, \alpha, \eta)+\beta P_{1}(\tau, \alpha, \beta, \eta), \\
& P^{\prime}(\tau, \alpha+i \beta, \eta)=P^{\prime}(\tau, \alpha, \eta)+\beta P_{1}^{\prime}(\tau, \alpha, \beta, \eta),
\end{aligned}
$$

where

$$
\begin{aligned}
& \left|P_{1}(\tau, \alpha, \beta, \eta)\right| \leqslant C(K)(|\tau|+|\xi|+|\eta|)^{m^{-1}}, \\
& \left|P_{1}^{\prime}(\tau, \alpha, \beta, \eta)\right| \leqslant C(K)(|\tau|+|\xi|+|\eta|)^{m^{\prime-1}} .
\end{aligned}
$$

Therefore we have

$$
\begin{aligned}
& |P(\tau, \xi, \eta)|^{n^{\prime}}+\left|P^{\prime}(\tau, \xi, \eta)\right|^{m} \\
\geqslant & \left(|P(\tau, \alpha, \eta)|^{n^{\prime}}+\left|P^{\prime}(\tau, \alpha, \eta)\right|^{m}\right)-C^{\prime}(K)|\beta|(|\tau|+|\xi|+|\eta|)^{m m-1},
\end{aligned}
$$

and then there exists $c^{\prime}=c^{\prime}\left(K, k_{1}\right)>0$ such that 


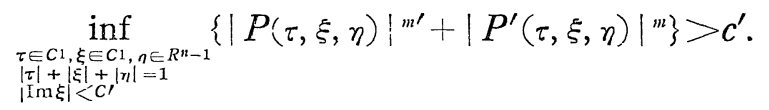

The converse is shown easily.

Lemma 2. 2. We assume (I) and (II). Then $P, P^{\prime}$ are decomposed into

$$
P=P_{0} P_{(-)}, \quad P^{\prime}=P_{0}^{\prime} P_{(-)}^{\prime},
$$

where

$$
\inf _{\substack{\operatorname{lin} \tau=0, \eta \in R^{n-1} \\|\tau|+|\eta|=1}}\left|R_{P_{0} P_{0}^{\prime}}(\tau, \eta)\right| \geqslant c^{\prime}
$$

all the roots of $P_{(-)}, P_{(-)}^{\prime}$ have negative imaginary parts, and

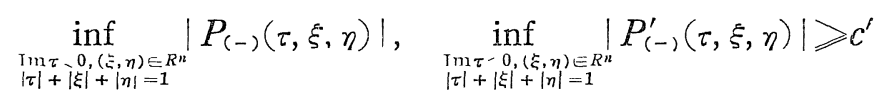

where $c^{\prime}=c^{\prime}\left(K, k_{1}, k_{2}\right)>0$.

Proof. From Lemma 2.1, we have the two cases for $\xi_{\jmath}(\tau, \eta)$ :

i) $\left|\operatorname{Im} \xi_{j}(\tau, \eta)\right| \geqslant c^{\prime}(|\tau|+|\eta|)$,

ii) $\left|\operatorname{Im} \xi_{j}(\tau, \eta)\right|<c^{\prime}(|\tau|+|\eta|)$ and $\left|P^{\prime}\left(\tau, \xi_{j}(\tau, \eta), \eta\right)\right| \geqslant c^{\prime}(|\tau|+|\eta|)^{m \prime}$, for $\operatorname{Im} \tau<0, \eta \in R^{n-1}$. The analogous situation holds for $\xi_{j}^{\prime}$. Now we denote

$$
\begin{aligned}
& P_{(0)}(\tau, \xi, \eta)=\prod_{\operatorname{Im} \xi^{-}(\tau, \eta)>-c^{\prime}(|\tau|+|\eta|)}\left(\xi-\xi_{j}^{-}(\tau, \eta)\right), \\
& P_{(-)}(\tau, \xi, \eta)=a_{0} \underset{\operatorname{Im} \xi^{-}(\tau, \eta) \leqslant-c^{\prime}(|\tau|+|\eta|)}{ }\left(\xi-\xi_{j}^{-}(\tau, \eta)\right), \\
& P_{0}(\tau, \xi, \eta)=P_{+}(\tau, \xi, \eta) P_{(0)}(\tau, \xi, \eta),
\end{aligned}
$$

where the degree of $P_{0}$ may vary with $(\tau, \eta)$. We define $P_{(0)}^{\prime}, P_{(-)}^{\prime}$, $P_{0}^{\prime}$ in the same way. Since we have

$$
R_{P_{0} P_{0}^{\prime}}=R_{P_{+} P_{+}^{\prime}} R_{P_{+} P_{(0)}^{\prime}} R_{P_{(0)} P_{+}^{\prime}} R_{P_{(0)} P_{(0)}^{\prime}}
$$

we have

$$
\inf _{\substack{\lim _{\tau-0, \eta \in R}|\tau|+|\eta|=1 \\|\tau|=1}}\left|R_{P_{0} P_{0}^{\prime}}(\tau, \eta)\right| \geqslant c\left(K, k_{1}, k_{2}\right)>0 .
$$

Now we denote

$$
\|u\|_{s, \tau}^{2}=\int_{R_{+}^{n}} \sum_{2+j+|\nu|=s}\left|\tau^{\imath} D_{x}^{j} D_{y}^{\nu} u(x, y)\right|^{2} d x d y .
$$


Proposition 2.1. We assume (I), then we have, for $u \in \mathscr{D}_{L^{2}}^{s}\left(R_{+}^{n}\right)$, $c_{s}\|u\|_{s, \tau} \leqslant\left\|P\left(\tau, D_{x}, D_{y}\right) u\right\|_{s-m, \tau}+\left\|P^{\prime}\left(\tau, D_{x}, D_{y}\right) u\right\|_{s-m^{\prime}, \tau}$ for $\operatorname{Im} \tau<0$, where $s \geqslant \max \left(m, m^{\prime}\right)$ and $c_{s}=c_{s}\left(K, k_{1}\right)>0$.

Proof. Remarking Lemma 2.1, we only apply Proposition 1.1.

Proposition 2.2. We assume (I) and (II), then we have, for $u \in \mathcal{E}_{L^{2}}^{s}\left(R_{+}^{n}\right)$,

$c_{s}\|u\|_{s, \tau} \leqslant\left\|P\left(\tau, D_{x}, D_{y}\right) u\right\|_{s-m, \tau}+\left\|P^{\prime}\left(\tau, D_{x}, D_{y}\right) u\right\|_{s-m^{\prime}, \tau}$ for $\operatorname{Im} \tau<0$, where $s \geqslant \max \left(m, m^{\prime}\right)$ and $c_{s}=c_{s}\left(K, k_{1}, k_{2}\right)>0$.

Proof. Remarking Lemma 2.2, we only apply Proposition 1.2.

Finally we consider the case of variable coefficients:

$$
\begin{aligned}
& P(x, y ; \tau, \xi, \eta)=\sum_{i+j+|\nu|=m} p_{i j \nu}(x, y) \tau^{i} \xi^{j} \eta^{\nu}, \\
& P^{\prime}(x, y ; \tau, \xi, \eta)=\sum_{i+j+|\nu|=m^{\prime}} p_{i j \nu}^{\prime}(x, y) \tau^{i} \xi^{j} \eta^{\nu},
\end{aligned}
$$

where $p_{i j \nu}(x, y), p_{i j \nu}^{\prime}(x, y) \in \mathscr{B}\left(R_{+}^{n}\right)$, and

$$
\inf _{(x, y) \in R_{+}^{n}}\left\{\left|p_{m 00}(x, y)\right|,\left|p_{0 m 0}(x, y)\right|,\left|p_{m \prime 00}^{\prime}(x, y)\right|,\left|p_{0 m^{\prime} 0}^{\prime}(x, y)\right|\right\} \neq 0 \text {. }
$$

Theorem 2.1. We assume that $P, P^{\prime}$ are hyperbolic with respect to $\tau$ for each point $(x, y) \in \mathbb{R}_{+}^{n}$, and

$$
\inf _{\substack{(x, y) \in R_{+}^{n} \\(\xi \xi \eta) \in R^{n} \\|\xi|+|\eta|=1}}\left|R_{P P^{\prime}}^{0}(x, y ; \xi, \eta)\right| \neq 0
$$

$$
\inf _{\substack{y \in R n-1 \\ y \in R^{n-1} \\ \tau m<0, \eta \in R^{n-1} \\|\tau|+|\eta|=1}}\left|R_{P P}^{+}(0, y ; \tau, \eta)\right| \neq 0 .
$$

Then we have for $u \in \mathcal{E}_{L^{2}}^{s}\left(R_{+}^{n}\right)$

$$
\left\|P\left(x, y ; \tau, D_{x}, D_{y}\right) u\right\|_{s-m, \tau}+\left\|P^{\prime}\left(x, y ; \tau, D_{x}, D_{y}\right) u\right\|_{s-m^{\prime}, \tau} \geqslant c_{s}\|u\|_{s, \tau}
$$

for $\operatorname{Im} \tau<0$ and $|\tau|>C_{s}$, where $s \geqslant \max \left(m, m^{\prime}\right)$ and $c_{s}, C_{s}$ are positive constants.

Proof. Using partition of unity in $R_{+}^{n}$, we apply Proposition 2.2 near the boundary of $R_{+}^{n}$ and Proposition 2.1 in the interior. 


\section{Lopatinski's determinants.}

Given a system of polynomials $\left\{A(\xi) ; B_{1}(\xi), \cdots, B_{m}(\xi)\right\}$, we define Lopatinski's determinant of $\left\{A ; B_{1}, \cdots, B_{m}\right\}$ by

$$
\operatorname{Lop}\left\{A ; B_{1}, \cdots, B_{m}\right\}=\operatorname{det}\left(\frac{1}{2 \pi i} \oint_{\Gamma} \frac{B_{i}(\xi) \xi^{m-j}}{A(\xi)} d \xi\right)_{i, j=1, \cdots, m},
$$

where $\Gamma$ is a simple closed curve enclosing all the roots of $A$.

Lemma 3.1. Let $A(\xi)=\prod_{j=1}^{m}\left(\xi-\xi_{j}\right)$, then

$$
\operatorname{Lop}\left\{A ; B_{1}, \cdots, B_{m}\right\}=\frac{1}{\prod_{i>j}\left(\xi_{i}-\xi_{j}\right)}\left|\begin{array}{c}
B_{1}\left(\xi_{1}\right) \cdots B_{1}\left(\xi_{m}\right) \\
\cdots \cdots \cdots \cdots \cdots \cdots \\
B_{m}\left(\xi_{1}\right) \cdots B_{m}\left(\xi_{m}\right)
\end{array}\right| .
$$

Moreover if $\left\{B_{1}, \cdots, B_{m}\right\}$ are given by

then

$$
\left(\begin{array}{c}
B_{1}(\xi) \\
\vdots \\
B_{m}(\xi)
\end{array}\right)=\left(\begin{array}{c}
b_{11} \cdots b_{1 m} \\
\cdots \cdots \cdots \cdots \\
b_{m 1} \cdots b_{m m}
\end{array}\right)\left(\begin{array}{c}
\xi^{m-1} \\
\vdots \\
1
\end{array}\right)=\mathscr{B}\left(\begin{array}{c}
\xi^{m-1} \\
\vdots \\
1
\end{array}\right)
$$

$$
\operatorname{Lop}\left\{A ; B_{1}, \cdots, B_{m}\right\}=(-1)^{\frac{1}{2} m(m-1)} \operatorname{det} \mathscr{B} .
$$

Remark. Let

then

$$
B_{j}(\xi)=C(\xi) C_{j}(\xi) \quad(j=1,2, \cdots, m)
$$

$$
\operatorname{Lop}\left\{A ; B_{1}, \cdots, B_{m}\right\}=\prod_{j=1}^{m} C\left(\xi_{j}\right) \operatorname{Lop}\left\{A ; C_{1}, \cdots, C_{m}\right\} .
$$

Proof.

$$
\begin{aligned}
& \operatorname{Lop}\left\{A ; B_{1}, \cdots, B_{m}\right\}=\operatorname{det}\left(\sum_{k} \frac{B_{i}\left(\xi_{k}\right) \xi_{k}^{m-j}}{\prod_{l \neq k}\left(\xi_{k}-\xi_{l}\right)}\right)_{i, j=1, \cdots, m} \\
& =\left|\begin{array}{c}
B_{1}\left(\xi_{1}\right) \cdots B_{1}\left(\xi_{m}\right) \\
\cdots \cdots \cdots \cdots \cdots \cdots \\
B_{m}\left(\xi_{1}\right) \cdots B_{m}\left(\xi_{m}\right)
\end{array}\right|\left|\begin{array}{ccc}
\frac{\xi_{1}^{m-1}}{\prod_{l \neq 1}\left(\xi_{1}-\xi_{l}\right)} \cdots \frac{1}{\prod_{l \neq 1}\left(\xi_{1}-\xi_{l}\right)} \\
\frac{\xi_{m}^{m-1}}{\prod_{l \dashv m}\left(\xi_{m}-\xi_{l}\right)} \cdots \cdots \cdots \cdots \cdots \cdots \cdots \cdots \cdots \cdots \cdots \cdots \cdots \cdots \\
\prod_{l \neq m}\left(\xi_{m}-\xi_{l}\right)
\end{array}\right| .
\end{aligned}
$$

If $\left(\begin{array}{c}B_{1} \\ \vdots \\ \dot{B}_{m}\end{array}\right)=\mathscr{B}\left(\begin{array}{c}\xi^{m-1} \\ \vdots \\ 1\end{array}\right), \quad \frac{1}{\prod_{i>j}\left(\xi_{i}-\xi_{j}\right)}\left|\begin{array}{c}B_{1}\left(\xi_{1}\right) \cdots B_{1}\left(\xi_{m}\right) \\ \cdots \cdots \cdots \cdots \cdots \cdots \\ B_{m}\left(\xi_{1}\right) \cdots B_{m}\left(\xi_{m}\right)\end{array}\right|$

$$
=\frac{1}{\prod_{i>j}\left(\xi_{i}-\xi_{j}\right)} \mid \begin{gathered}
b_{11} \cdots b_{1 m} \\
\cdots \cdots \cdots \\
b_{m 1} \cdots \\
\cdots
\end{gathered}
$$


Lemma 3.2. Let

$$
A(\xi)=\prod_{j=1}^{m}\left(\xi-\xi_{j}\right), \quad A^{\prime}(\xi)=\prod_{j=1}^{m \prime}\left(\xi-\xi_{j}^{\prime}\right),
$$

and let $\left\{B_{1}^{\prime}(\xi), \cdots, B_{m \prime}^{\prime}(\xi)\right\}$ be divisible by $A(\xi)$. Then

$$
\begin{aligned}
& \operatorname{Lop}\left\{A ; B_{1}, \cdots, B_{m}\right\} \operatorname{Lop}\left\{A^{\prime} ; B_{1}^{\prime}, \cdots, B_{m^{\prime}}^{\prime}\right\} \\
& \quad=(-1)^{m m^{\prime}} R_{A A^{\prime}} \operatorname{Lop}\left\{A A^{\prime} ; B_{1}, \cdots, B_{m}, B_{1}^{\prime}, \cdots, B_{m^{\prime}}^{\prime}\right\} .
\end{aligned}
$$

Remark. Let

then

$$
B_{j}^{\prime}(\xi)=A(\xi) C_{j}^{\prime}(\xi) \quad\left(j=1, \cdots, m^{\prime}\right)
$$

$$
\begin{aligned}
\operatorname{Lop}\left\{A ; B_{1},\right. & \left.\cdots, B_{m}\right\} \cdot \operatorname{Lop}\left\{A^{\prime} ; C_{1}^{\prime}, \cdots, C_{m^{\prime}}^{\prime}\right\} \\
& =\operatorname{Lop}\left\{A A^{\prime} ; B_{1}, \cdots, B_{m}, B_{1}^{\prime}, \cdots, B_{m^{\prime}}^{\prime}\right\} .
\end{aligned}
$$

Proof. Since $B_{i}^{\prime}\left(\xi_{j}\right)=0 \quad\left(i=1, \cdots, m^{\prime}, j=1, \cdots, m\right)$, we have from Lemma 3.1

$$
\begin{aligned}
\operatorname{Lop} & \left\{A A^{\prime} ; B_{1}, \cdots, B_{m}, B_{1}^{\prime}, \cdots, B_{m^{\prime}}^{\prime}\right\} \\
= & \frac{\operatorname{det}\left(B_{i}\left(\xi_{j}\right)\right)_{i, j=1, \cdots, m} \operatorname{det}\left(B_{i}^{\prime}\left(\xi_{j}^{\prime}\right)\right)_{i, j=1, \cdots, m}}{\prod_{i>j}\left(\xi_{i}-\xi_{j}\right) \prod_{i, j}\left(\xi_{i}^{\prime}-\xi_{j}\right) \prod_{i>j}\left(\xi_{i}^{\prime}-\xi_{j}^{\prime}\right)} \\
= & \frac{(-1)^{m \prime}}{R_{A A^{\prime}}} \operatorname{Lop}\left\{A ; B_{1}, \cdots, B_{m}\right\} \operatorname{Lop}\left\{A^{\prime} ; B_{1}^{\prime}, \cdots, B_{m^{\prime}}^{\prime}\right\} .
\end{aligned}
$$

Hereafter we consider $\left\{A ; B_{1}, \cdots, B_{\mu}\right\}$ in the following situations: Let

$$
\begin{aligned}
& A(\xi)=\xi^{m}+a_{1} \xi^{m-1}+\cdots+a_{m}=A_{+}(\xi) A_{-}(\xi), \\
& A_{+}(\xi)=\prod_{j=1}^{\mu}\left(\xi-\xi_{j}^{+}\right), \quad A_{-}(\xi)=\prod_{j=1}^{m-\mu}\left(\xi-\xi_{j}^{-}\right),
\end{aligned}
$$

where $\left\{\xi_{j}^{+}\right\}_{j=1, \cdots, \mu}$ have positive imaginary parts, $\left\{\xi_{j}^{-}\right\}_{j=1, \cdots, m-\mu}$ have negative ones and

$$
\begin{aligned}
& \left|a_{j}\right| \leqslant C r^{j} \quad j=1, \cdots, m, \\
& \left|A_{+}(\xi)\right| \geqslant c(|\xi|+r)^{\mu} \quad \text { for } \quad \xi \in R^{1}, \\
& \left|A_{-}(\xi)\right| \geqslant c(|\xi|+r)^{m-\mu} \quad \text { for } \quad \xi \in R^{1} .
\end{aligned}
$$

And let

where

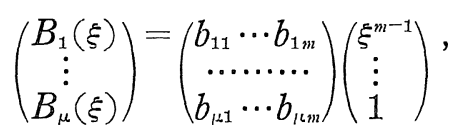

$$
\left|b_{i j}\right| \leqslant C r^{r_{i}-(m-j)}
$$


Proposition 3.1. We assume that

$$
\left|\operatorname{Lop}\left\{A_{+} ; B_{1}, \cdots, B_{\mu}\right\}\right| \geqslant d r^{r_{1}+\cdots+r_{\mu}-\frac{1}{2} \mu(u-1)},
$$

then we have for $u \in \mathcal{E}_{L^{2}}^{s}(0, \infty)$

$$
c_{s} \int_{0}^{\infty} \sum_{j=0}^{s}\left|r^{s-j} D^{j} u(x)\right|^{2} d x \leqslant \int_{0}^{\infty} \sum_{j=0}^{s-m}\left|r^{s-m-j} D^{j} A(D) u\right|^{2} d x+\sum_{j=1}^{\mu}\left|r^{s-r j-\frac{1}{2}} B_{j}(\omega)\right|^{2},
$$

where $s \geqslant m, c_{s}=c_{s}(C, c, d)>0$.

Proof. Let us denote

$$
B_{k}(\xi)=\xi^{k-\mu-1} A_{+}(\xi) \quad(k=\mu+1, \cdots, m), \quad\left(\begin{array}{c}
B_{1} \\
\vdots \\
B_{m}
\end{array}\right)=\mathscr{B}\left(\begin{array}{c}
\xi^{m-1} \\
\vdots \\
1
\end{array}\right),
$$

then we have from Lemma 3.1

$$
\begin{aligned}
\operatorname{Lop}\left\{A_{-} ; B_{\mu+1}, \cdots, B_{m}\right\}=\frac{1}{\prod_{i>j}\left(\xi_{i}^{-}-\xi_{j}^{-}\right)}\left|\begin{array}{cc}
A_{+}\left(\xi_{1}^{-}\right) \cdots & A_{+}\left(\xi_{m-\mu}^{-}\right) \\
\xi_{1}^{-} A_{+}\left(\xi_{1}^{-}\right) \cdots \xi_{m-\mu}^{-} A_{+}\left(\xi_{m-\mu}^{-}\right) & \cdots \cdots \cdots \cdots \cdots \cdots \cdots \cdots \cdots \cdots \cdots \cdots \cdots
\end{array}\right| \\
=A_{+}\left(\xi_{1}^{-}\right) \cdots A_{+}\left(\xi_{m-\mu}^{-}\right)=(-1)^{\mu(m-\mu)} R_{A_{+} A_{-}}
\end{aligned}
$$

and

$$
\operatorname{Lop}\left\{A ; B_{1}, \cdots, B_{m}\right\}=(-1)^{\frac{1}{2} m(m-1)} \operatorname{det} \mathscr{B} .
$$

Therefore we have from Lemma 3.2

$$
\operatorname{Lop}\left\{A_{+} ; B_{1}, \cdots, B_{l}\right\}=(-1)^{\mu(m-\mu)+\frac{1}{2} m(m-1)} \operatorname{det} \mathscr{B} .
$$

Now let

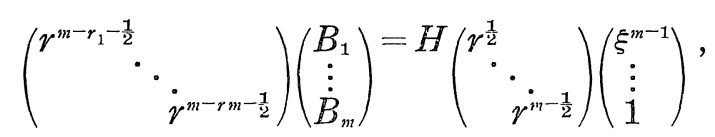

then we have $|\operatorname{det} H| \geqslant d$, and then every elements of $H^{-1}$ are bounded by $C^{\prime}(C, d)$. Therefore we have

$$
\sum_{j=1}^{m} r^{j-\frac{1}{2}}\left|\omega_{m-j}\right| \leqslant C^{\prime}(C, d) \sum_{j=1}^{m} r^{m-r,-\frac{1}{2}}\left|B_{j}(\omega)\right| .
$$

On the other hand, since

$$
r\left|B_{j}(\omega)\right|^{2} \leqslant \int_{0}^{\infty}\left(\left|D B_{j}(D) u(x)\right|^{2}+r^{2}\left|B_{j}(D) u(x)\right|^{2}\right) d x,
$$

we have

$$
\sum_{j=\mu+1}^{m} r^{2\left(m-r,-\frac{1}{2}\right)}\left|B_{j}(\omega)\right|^{2} \leqslant \sum_{j=\mu+1}^{m} r^{2(m-r,-1)}\left\{\int _ { 0 } ^ { \infty } \left(\left|D^{j-\mu} A_{+}(D) u(x)\right|^{2}\right.\right.
$$




$$
\left.\left.+r^{2}\left|D^{j-\mu-1} A_{+}(D) u(x)\right|^{2}\right) d x\right\} \leqslant 2 \sum_{j=0}^{m-\mu} r^{2(m-\mu-j)} \int_{0}^{\infty}\left|D^{j} A_{+}(D) u(x)\right|^{2} d x .
$$

Then we have from Lemma 1.3

$$
\begin{aligned}
c_{0}(C, c, d) \int_{0}^{\infty} \sum_{j=1}^{m}\left|r^{m-j} D^{j} u(x)\right|^{2} d x \leqslant \int_{0}^{\infty}|A(D) u(x)|^{2} d x \\
+\sum_{j=1}^{\mu}\left|r^{m-r,-\frac{1}{2}} B_{j}(\omega)\right|^{2}+\int_{0}^{\infty} \sum_{j=0}^{m-\mu}\left|r^{m-\mu-j} D^{j} A_{+}(D) u(x)\right|^{2} d x,
\end{aligned}
$$

and we have from Lemma 1.5

$$
c_{1}(C, c) \int_{0}^{\infty} \sum_{j=0}^{m-\mu}\left|r^{m-\mu-j} D^{j} A_{+}(D) u(x)\right|^{2} d x \leqslant \int_{0}^{\infty}|A(D) u(x)|^{2} d x .
$$

For $s>m$, we put $A M^{s-m}$ instead of $A$.

Next we consider an adjoint system of $\left\{A ; B_{1}, \cdots, B_{\mu}\right\}$. Let us denote

$$
\begin{aligned}
& \bar{A}(\xi)=\xi^{m}+\bar{a}_{1} \xi^{m-1}+\cdots+\bar{a}_{m},
\end{aligned}
$$

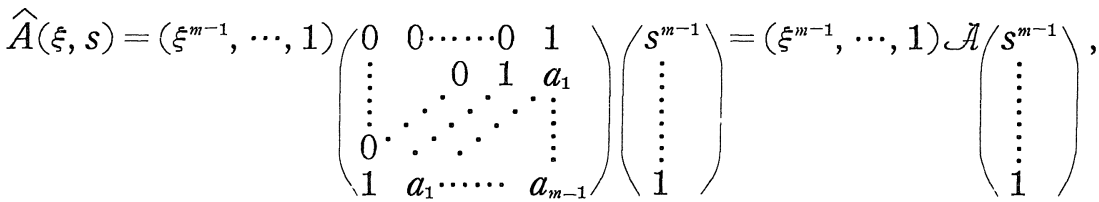

and

$$
(u, v)=\int_{0}^{\infty} u(x) \overline{v(x)} d x
$$

Since

$$
\begin{gathered}
\left(D^{k} u, v\right)=i\left\{D^{k-1} u(0) \overline{v(0)}+D^{k-2} u(0) \overline{D v(0)}+\cdots+u(0) \overline{D^{k-1} v(0)}\right\} \\
+\left(u, D^{k} v\right)
\end{gathered}
$$

we have

$$
(A(D) u, v)-(u, \bar{A}(D) v)=i \overline{\left(D^{m-1} v(0), \cdots, v(0)\right)} \AA\left(\begin{array}{c}
D^{m-1} u(0) \\
\vdots \\
u(0)
\end{array}\right)
$$

Now we assume that

$$
\operatorname{Lop}\left\{A_{+} ; B_{1}, \cdots, B_{\mu}\right\} \neq 0,
$$

which implies that $\operatorname{det} \mathscr{B} \neq 0$, then we denote

$$
\left(\begin{array}{c}
B_{1}^{\prime}(\xi) \\
\vdots \\
B_{m}^{i}(\xi)
\end{array}\right)=\left(\mathscr{B}^{-1}\right) * \mathscr{A}^{*}\left(\begin{array}{c}
\xi^{m-1} \\
\vdots \\
1
\end{array}\right)
$$

Here we have 


\section{Proposition 3.2. We assume that}

$$
\left|\operatorname{Lop}\left\{A_{+} ; B_{1}, \cdots, B_{\mu}\right\}\right| \geqslant d r^{r_{1}+\cdots+r_{\mu}-\frac{1}{2} \mu(\mu-1)},
$$

then there exists a system of polynomials $\left\{B_{j}, B_{j}^{\prime}\right\}_{j=1, \cdots, m}$, and it satisfies that

$$
(A(D) u, v)-(u, \bar{A}(D) v)=i \sum_{j=1}^{m} B_{j} u(0) \overline{B_{j}^{\prime} v(0)} \text { for } u, v \in \mathcal{E}_{L^{2}}^{m}(0, \infty),
$$

and

$$
\left|\operatorname{Lop}\left\{\bar{A}_{-} ; B_{\mu+1}^{\prime}, \cdots, B_{m}^{\prime}\right\}\right| \geqslant c_{0}(C, d)>0 .
$$

Proof. To show the last statement, let

$$
u(x)=e^{i \xi_{t} x}, \quad v(x)=e^{i \overline{\xi_{\bar{I}}} x},
$$

then we have

$$
\sum_{j=1}^{m} B_{j}\left(\xi_{k}^{+}\right) \overline{B_{j}^{\prime}\left(\overline{\xi_{l}^{-}}\right)}=0
$$

that is,

$$
\left(\begin{array}{c}
B_{1}\left(\xi_{1}^{+}\right) \cdots B_{m}\left(\xi_{1}^{+}\right) \\
\cdots \cdots \cdots \cdots \cdots \cdots \\
B_{1}\left(\xi_{\mu}^{+}\right) \cdots B_{m}\left(\xi_{\mu}^{+}\right)
\end{array}\right)\left(\begin{array}{c}
\overline{B_{1}^{\prime}\left(\overline{\xi_{1}^{-}}\right) \cdots B_{1}^{\prime}\left(\overline{\xi_{m}}\right)} \\
\cdots \cdots \cdots \cdots \cdots \cdots \cdots \\
B_{m}^{\prime}\left(\overline{\xi_{1}^{-}}\right) \cdots B_{m}^{\prime}\left(\overline{\xi_{m}^{-}}\right)
\end{array}\right)=0 .
$$

Since $B_{k}\left(\xi_{1}^{+}\right)=\cdots=B_{k}\left(\xi_{\mu}^{+}\right)=0(k=\mu+1, \cdots, m)$, we have

$$
B_{k}^{\prime}\left(\overline{\xi_{1}^{-}}\right)=\cdots=B_{k}^{\prime}\left(\overline{\xi_{m-\mu}^{-}}\right)=0 \quad(k=1, \cdots, \mu),
$$

that is, $\left\{B_{1}^{\prime}(\xi), \cdots, B_{\mu}^{\prime}(\xi)\right\}$ are all divisible by $\overline{A_{-}}(\xi)$. Now let

$$
B_{j}^{\prime}(\xi)=\bar{A}_{-}(\xi) C_{j}^{\prime}(\xi) \quad(j=1, \cdots, \mu),
$$

where the absolute values of the coefficients of $\left\{C_{j}^{\prime}(\xi)\right\}$ are bounded by a positive constant $K=K(C, d)$ if $r=1$, then we have from Lemma 3. 1 and Lemma 3. 2

$$
\begin{aligned}
& \operatorname{Lop}\left\{\overline{A_{+}} ; C_{1}^{\prime}, \cdots, C_{\mu}^{\prime}\right\} \operatorname{Lop}\left\{\overline{A_{-}} ; B_{\mu+1}^{\prime}, \cdots, B_{m}^{\prime}\right\} \\
& \quad=\operatorname{Lop}\left\{\bar{A} ; B_{\mu+1}^{\prime}, \cdots, B_{m}^{\prime}, B_{1}^{\prime}, \cdots, B_{\mu}^{\prime}\right\}=(-1)^{\mu(m-\mu)} \operatorname{Lop}\left\{\bar{A} ; B_{1}^{\prime}, \cdots, B_{m}^{\prime}\right\} \\
& \quad=(-1)^{\mu(m-\mu)}(-1)^{\frac{1}{2 m(m-1)}} \operatorname{det}\left(\left(\mathscr{B}^{-1}\right)^{*} \mathcal{A}^{*}\right)=(-1)^{\mu(m-\mu)} \overline{(\operatorname{det} \mathscr{B})^{-1}} .
\end{aligned}
$$

Finally we consider equivalent boundary conditions. We say that $\left\{B_{1}(\xi), \cdots, B_{\mu}(\xi)\right\}$ and $\left\{\widetilde{B}_{1}(\xi), \cdots, \widetilde{B_{\mu}}(\xi)\right\}$ are equivalent, if the condition $\left\{\left.B_{j}(D) u\right|_{x=0}=0(j=1, \cdots, \mu)\right\}$ is equivalent to the condition $\left\{\left.B_{j}(D) u\right|_{x=0}\right.$ 
$=0(j=1, \cdots, \mu)\}$ for $u \in C^{\infty}\left(R^{1}\right)$.

Lemma 3. 3. Let $\left\{B_{j}(\xi)\right\}_{j=1, \cdots, \mu}$ and $\left\{\widetilde{B}_{j}(\xi)\right\}_{j=1, \cdots, \mu}$ be sets consisting of linearly independent polynomials of order less than $m$. Then, in order that $\left\{B_{j}(\xi)\right\}$ and $\left\{\widetilde{B_{j}}(\xi)\right\}$ are equivalent, it is necessary and sufficient that there exists a regular constant matrix

such that

$$
\mathcal{C}=\left(\begin{array}{ccc}
C_{11} & \cdots & C_{1 \mu} \\
\vdots & & \vdots \\
C_{\mu 1} & \cdots & C_{\mu l}
\end{array}\right)
$$

$$
\left(\begin{array}{c}
B_{1}(\xi) \\
\vdots \\
B_{\mu}(\xi)
\end{array}\right)=C\left(\begin{array}{c}
\widetilde{B_{1}}(\xi) \\
\vdots \\
\widetilde{B}_{\mu}(\xi)
\end{array}\right) \cdot
$$

Proof. Let us denote

$$
B_{j}(\xi)=\sum_{k=1}^{m} b_{j k} \xi^{m-k}, \quad \widetilde{B_{j}}(\xi)=\sum_{k=1}^{m} \widetilde{b_{j k}} \xi^{m-k} \quad(j=1, \cdots, \mu) .
$$

Then, in order that $\left\{B_{j}(\xi)\right\}$ and $\left\{\widetilde{B}_{j}(\xi)\right\}$ are equivalent, it is necessary and sufficient that

$$
\left(\begin{array}{ccc}
b_{11} & \cdots & b_{1 n} \\
\vdots & \vdots \\
b_{\mu 1} & \cdots & b_{\mu m}
\end{array}\right)\left(\begin{array}{c}
\omega_{m-1} \\
\vdots \\
\omega_{0}
\end{array}\right)=0
$$

is equivalent to

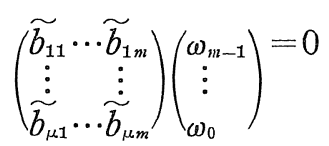

for $\left(\omega_{m-1}, \cdots, \omega_{0}\right) \in C^{m}$, that is, the space generated by $\left\{b_{j}=\left(b_{j 1}, \cdots\right.\right.$, $\left.\left.b_{j m}\right)\right\}_{j=1, \cdots, \mu}$ is equal to the space generated by $\left\{\widetilde{b}_{j}=\left(\widetilde{b}_{j 1}, \cdots, \widetilde{b}_{j m}\right)\right\}_{j=1, \cdots, \mu}$. Hence we have

$$
b_{j}=\sum_{k=1}^{\mu} c_{j k} \widetilde{b_{k}} \quad j=1, \cdots, \mu .
$$

Corollary。 Let $\widetilde{B}_{j}(\xi)$ be a normal set such that

$$
\widetilde{b}_{j 1}=\cdots=\widetilde{b}_{m_{m-s_{j}-1}}=0, \widetilde{b}_{j-s_{j}}=1 \quad(j=1, \cdots, \mu),
$$

and

and let

$$
s_{i} \neq s_{j} \quad \text { if } \quad i \neq j,
$$




$$
\left|b_{j k}\right| \leqslant C_{1}, \quad\left|\widetilde{b}_{j k}\right| \leqslant C_{2} \quad(j=1, \cdots, \mu, k=1, \cdots, m) .
$$

Then there exists a positive number $\delta=\delta\left(C_{1}, C_{2}\right)$ such that

$$
\left|\operatorname{Lop}\left\{A_{+} ; \widetilde{B}_{1}, \cdots, \widetilde{B}_{\mu}\right\}\right| \geqslant \delta\left|\operatorname{Lop}\left\{A_{+} ; B_{1}, \cdots, B_{\mu}\right\}\right| .
$$

Proof. Let us assume $s_{1}>s_{2}>\cdots>s_{\mu}$ without loss of generality. From the Lemma 3.3, we have

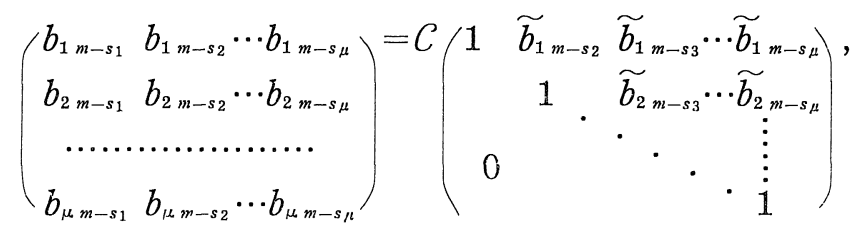

therefore we have

$$
\left|c_{i j}\right| \leqslant K\left(C_{1}, C_{2}\right) \quad(i, j=1, \cdots, \mu),
$$

hence

$$
|\operatorname{det} \mathcal{C}| \leqslant K_{1}\left(C_{1}, C_{2}\right) \text {. }
$$

On the other hand, we have

$$
\operatorname{Lop}\left\{A_{+} ; B_{1}, \cdots, B_{\mu}\right\}=\operatorname{det} C \cdot \operatorname{Lop}\left\{A_{+} ; \widetilde{B}_{1}, \cdots, \widetilde{B}_{\mu}\right\},
$$

then

$$
\left|\operatorname{Lop}\left\{A_{+} ; \widetilde{B}_{1}, \cdots, \widetilde{B}_{\mu}\right\}\right| \geqslant \frac{1}{K_{1}\left(C_{1}, C_{2}\right)}\left|\operatorname{Lop}\left\{A_{+} ; B_{1}, \cdots, B_{\mu}\right\}\right| .
$$

\section{4. $\lambda$-elliptic $(\lambda<0)$ operator in $\mathbb{R}_{+}^{n}$}

Let us consider

$$
L(x, y ; \lambda, \xi, \eta)=\left\{A(x, y ; \lambda, \xi, \eta) ; B_{1}(y ; \lambda, \xi, \eta), \cdots, B_{m}(y ; \lambda, \xi, \eta)\right\},
$$

where $\left\{A, B_{1}, \cdots, B_{m}\right\}$ are homogeneous polynomials of degree $\left\{2 m, r_{1}\right.$, $\left.\cdots, r_{n}\right\}$ with respect to $(\lambda, \xi, \eta)$ :

$$
\begin{aligned}
& A=\xi^{2 m}+a_{1}(x, y ; \lambda, \eta) \xi^{2 m-1}+\cdots+a_{2 m}(x, y ; \lambda, \eta), \\
& B_{j}=\xi^{r_{j}}+b_{j m-r, 1}(y ; \lambda, \eta) \xi^{r_{j}-1}+\cdots+b_{j m}(y ; \lambda, \eta),
\end{aligned}
$$

where the coefficients of $A$ and $B_{j}$ belong to $\mathscr{B}\left(R_{+}^{n}\right)$.

Now we assume that

$$
\inf _{\substack{x, y) \in R_{+}^{n} \\ \text { and } \\ \text { and } 0,(y, \eta) \in R^{n} \\|\lambda|+|\xi|+|\eta|-1}}|A(x, y ; \lambda, \xi, \eta)| \neq 0,
$$


then we can decompose

$$
A=A_{+} A_{-}=\prod_{j=1}^{m}\left(\xi-\xi_{j}^{+}(x, y ; \lambda, \eta)\right) \prod_{j=1}^{m}\left(\xi-\xi_{j}^{-}(x, y ; \lambda, \eta)\right) .
$$

We denote Lopatinski's determinant of $\left\{A_{+}(0, y ; \lambda, \xi, \eta) ; B_{1}(y ; \lambda, \xi, \eta)\right.$, $\left.\cdots, B_{m}(y ; \lambda, \xi, \eta)\right\}$ with respect to $\xi$ by $\operatorname{Lop}\left\{A_{+} ; B_{1}, \cdots, B_{m}\right\}(y ; \lambda, \eta)$. We say that $L$ is $\lambda$-elliptic $(\lambda>0)$ in the half space $R_{+}^{n}$, if

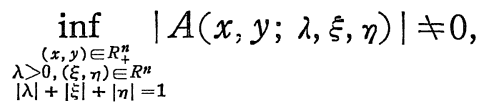

and

$$
\inf _{\substack{y \in R^{n-1} \\ y=, n \in R-1 \\ \lambda|\lambda|+|\eta|=1}}\left|\operatorname{Lop}\left\{A_{+} ; B_{1}, \cdots, B_{m}\right\}(y ; \lambda, \eta)\right| \neq 0
$$

We denote

$$
\begin{aligned}
& L\left(x, y ; \lambda, D_{x}, D_{y}\right) u(x, y)=\left\{A\left(x, y ; \lambda, D_{x}, D_{y}\right) u(x, y) ;\right. \\
& \left.B_{1}\left(y ; \lambda, D_{x}, D_{y}\right) u(0, y), \cdots, B_{m}\left(y ; \lambda, D_{x}, D_{y}\right) u(0, y)\right\},
\end{aligned}
$$

then $L$ is a bounded operator from $\mathcal{E}_{L 2}^{s+2 m}\left(R_{+}^{n}\right)$ to

$$
\mathcal{E}_{L^{2}}^{s}\left(R_{+}^{n}\right) \times \prod_{j=}^{m} \mathcal{E}_{L^{2}}^{s+2 m-r_{j}-\frac{1}{2}}\left(R^{n-1}\right)\left(=\mathfrak{E}_{s}\right) .
$$

Let $F=\left\{f ; g_{1}, \cdots, g_{m}\right\}$ belong to $\mathfrak{S}_{s}$, we introduce norms of $F$ in $\mathfrak{L}_{s}$ by

$$
\|F\|_{s, \lambda}=\|f\|_{s, \lambda}+\sum_{j=1}^{m}\left\langle g_{j}\right\rangle_{s+2 m-r_{j}-\frac{1}{2}, \lambda},
$$

where $s$ is a non-negative integer, $\alpha$ is a real number, and

$$
\begin{aligned}
& \|f\|_{s, \lambda}^{2}=\int_{R_{+}^{n}} \sum_{i+j+|\nu|=s}\left|\lambda^{j} D_{x}^{j} D_{y}^{\nu} f(x, y)\right|^{2} d x d y, \\
& \langle g\rangle_{\alpha, \lambda}^{2}=\int_{R^{n-1}}\left(|\lambda|^{2}+|\eta|^{2}\right)^{\alpha}|\hat{g}(\eta)|^{2} d \eta,
\end{aligned}
$$

where

$$
g(\eta)=\int_{R-} e^{-i y^{\eta}} g(y) d y
$$

Theorem 4.1. We assume that $L$ is $\lambda$-elliptic $(\lambda>0)$ in $R_{+}^{n}$, then we have

$$
\|u\|_{2 m+s, \lambda} \leqslant C_{s}\left(\|L u\|_{s, \lambda}+\|u\|\right) \quad \text { for } \lambda>0,
$$

where $u \in \mathcal{E}_{L 2}^{2 m+s}\left(R_{+}^{n}\right)(s \geqslant 0)$. 
Proof. Using partition of unity in $R_{+}^{n}$, we apply Proposition 3.1 near the boundary of $R_{+}^{n}$ and Proposition 2.1 in the interior.

Let us say that $L u=F$ is solvable at $\lambda$, if for any $F \in \mathfrak{S}_{0}$ there exists a unique solution $u \in \mathcal{E}_{L_{2}}^{2 m}\left(R_{+}^{n}\right)$.

Theorem 4.2. We assume that $L$ is $\lambda$-elliptic $(\lambda>0)$ in $R_{+}^{n}$, then $L u=F$ is solvable for $\lambda>\lambda_{0}$, where $\lambda_{0}$ is a positive constant.

The proof is given in the appendix.

\section{§2. Iterated Hyperbolic Mixed Problems}

\section{Hy perbolic energy.}

Given a system of $m+1$ homogeneous polynomials with respect to $(\tau, \xi, \eta)$ :

$$
\begin{gathered}
L(x, y ; \tau, \xi, \eta)=\left\{A(x, y ; \tau, \xi, \eta) ; B_{1}(y ; \tau, \xi, \eta), \cdots, B_{n}(y ; \tau, \xi, \eta)\right\} \\
\left(x>0, y \in R^{n-1}\right),
\end{gathered}
$$

whose degrees are $\left\{2 m ; r_{1}, \cdots, r_{m}\right\} \quad\left(0 \leqslant r_{j} \leqslant 2 m-1\right)$. We say that $L$ is $\tau$-elliptic $(\operatorname{Im} \tau<0)$, if $L\left(x, y ; \lambda e^{-i \theta}, \xi, \eta\right)$ is $\lambda$-elliptic $(\lambda>0)$ for each $(0<\theta<\pi)$. We say that $L$ has the energy inequality of hyperbolic type, if

$$
\|u\|_{2 m-1, \tau} \leqslant \frac{C}{|\operatorname{Im} \tau|}\|L u\|_{0, \tau} \text { for } \operatorname{Im} \tau<-\gamma_{0}\left(\gamma_{0}>0\right)
$$

Now we consider

$$
L_{l}(x, y ; \tau, \xi, \eta)=\left\{A_{l}(x, y ; \tau, \xi, \eta) ; B_{l 1}(y ; \tau, \xi, \eta), \cdots, B_{l m_{l}}(y ; \tau, \xi, \eta)\right\}
$$

$(l=1,2, \cdots, N)$, where the degree of $A_{l}$ is $2 m_{l}$ and the degree of $B_{l j}$ is $r_{l j}$. Here we assume that

i) each $L_{l}$ is $\tau$-eiliptic $(\operatorname{Im} \tau<0)$,

ii) each $L_{l}$ has tise energy inequality of hyperbolic type, iii)

$$
\text { (I) } \inf _{\substack{(x, y) \in R_{n}^{n} \\(\xi, \eta) \in R^{n} \\|\xi|+|\eta|=1}}\left|\prod_{i<j} R_{A_{\mathfrak{s}} A_{j}}^{0}(x, y ; \xi, \eta)\right| \neq 0 \text {, }
$$


(II) $\quad \inf _{\substack{y \in R^{n-1} \\ \operatorname{Im} \tau<0, \eta \in R^{n-1} \\|\tau|+|\eta|=1}}\left|\prod_{i<j} R_{A_{i} A}^{+}(0, y ; \tau, \eta)\right| \neq 0$.

Now we denote

$$
\begin{aligned}
& A(x, y ; \tau, \xi, \eta)=\prod_{l=1}^{N} A_{l}(x, y ; \tau, \xi, \eta), \\
& Q_{k}(x, y ; \tau, \xi, \eta)=\prod_{l \neq k} A_{l}(x, y ; \tau, \xi, \eta) \quad(k=1,2, \cdots, N), \\
& B_{m_{1}+\cdots+m_{l-1+k}}(x, y ; \tau, \xi, \eta)=B_{l k}(y ; \tau, \xi, \eta) Q_{l}(x, y ; \tau, \xi, \eta) \\
& \left(k=1,2, \cdots, m_{l}, l=1,2, \cdots, N\right),
\end{aligned}
$$

and

$$
\begin{aligned}
& L(x, y ; \tau, \xi, \eta) \\
= & \left\{A(x, y ; \tau, \xi, \eta) ; B_{1}(0, y ; \tau, \xi, \eta), \cdots, B_{m}(0, y ; \tau, \xi, \eta)\right\},
\end{aligned}
$$

where $m=m_{1}+\cdots+m_{N}$ and $r_{m_{1+} \cdot+m_{l-1+k}}=2\left(m-m_{l}\right)+r_{l k}$.

Lemma 1. 1.

$\operatorname{Lop}\left\{A_{+} ; B_{1}, \cdots, B_{m}\right\}=\prod_{i<j} R_{A_{i}^{+} A_{j}^{+}} \prod_{i \neq j} R_{A_{i}^{+} A_{j}^{-}} \prod_{l=1}^{N} \operatorname{Lop}\left\{A_{l}^{+} ; B_{l 1}, \cdots, B_{l m_{l}}\right\}$.

Proof. Let's denote for $k=2,3, \cdots, N$

$$
\begin{aligned}
& Q_{1}^{(k)}=A_{2} A_{3} \cdots A_{k}, \\
& Q_{2}^{(k)}=A_{1} A_{3} \cdots A_{k}, \\
& \quad \cdots \cdots \cdots \cdots \\
& Q_{k}^{(k)}=A_{1} A_{2} \cdots A_{k-1}, \\
& A^{(k)}=A_{1} A_{2} \cdots A_{k}\left(=Q_{k+1}^{(k+1)}\right), \\
& \left\{B_{1}^{(k)}, B_{2}^{(k)}, \cdots, B_{m_{1}+\cdots+m_{k}}^{(k)}\right\}=\left\{B_{11} Q_{1}^{(k)}, \cdots, B_{1 m_{1}} Q_{1}^{(k)}, \cdots\right. \\
& \left.\quad \cdots, B_{k 1} Q_{k}^{(k)}, \cdots, B_{k m_{k}} Q_{k}^{(k)}\right\},
\end{aligned}
$$

then

$Q_{j}^{(N)}=Q_{j}(j=1, \cdots, N), \quad\left\{A^{(N)} ; B_{1}^{(N)}, \cdots, B_{m}^{(N)}\right\}=\left\{A ; B_{1}, \cdots, B_{m}\right\}$.

Now we assume that

$$
\begin{aligned}
& \operatorname{Lop}\left\{A^{(k)} ; B_{1}^{(k)}, \cdots, B_{m_{1}+\cdots+n_{k}}^{(k)}\right\} \\
& \quad=\prod_{\imath \leq j \leqslant k} R_{A_{i}^{+} A_{j}^{+}} \prod_{\substack{i \neq j \\
i j, \leqslant k}} R_{A_{i}^{+} A_{j}^{-}} \prod_{l=1}^{k} \operatorname{Lop}\left\{A_{l}^{+} ; B_{l 1}, \cdots, B_{l m}\right\} .
\end{aligned}
$$

On the other hand, we have 


$$
\begin{aligned}
& \operatorname{Lop}\left\{A_{+}^{(k)} ; B_{1}^{(k+1)}, \cdots, B_{n_{1}+\cdots+m_{k}}^{(k+1)}\right\} \\
= & \operatorname{Lop}\left\{A_{+}^{(k)} ; B_{1}^{(k)} A_{k+1}, \cdots, B_{m_{1}+\cdots+m_{k}}^{(k)} A_{k+1}\right\} \\
= & R_{A_{+}^{(k)} A_{k+1}} \operatorname{Lop}\left\{A_{+}^{(k)} ; B_{1}^{(k)}, \cdots, B_{m_{1}+\cdots+m_{k}}^{(k)}\right\}, \\
& \operatorname{Lop}\left\{A_{k+1}^{+} ; B_{m_{1}+\cdots+m_{k}+1}^{(k+1)} \cdots, B_{m_{1}+\cdots+m_{k+1}}^{(k+1)}\right\} \\
= & \operatorname{Lop}\left\{A_{k+1}^{+} ; B_{k+11} A^{(k)}, \cdots, B_{k+1 m_{k+1}} A^{(k)}\right\} \\
= & R_{A_{k+1} A^{(k)}} \operatorname{Lop}\left\{A_{k+1}^{+} ; B_{k \mid 11}, \cdots, B_{k+1}{ }_{m k+1}\right\},
\end{aligned}
$$

and we have from Lemma 3.2 in $\$ 1$.

$$
\begin{gathered}
\operatorname{Lop}\left\{A_{+}^{(k)} ; B_{1}^{(k+1)}, \cdots, B_{m_{+}+\cdots+m_{k}}^{(k+1)} \operatorname{Lop}\left\{A_{k+1}^{+} ; B_{m_{1}+\cdots+m_{k}+1}^{(k+1)}, \cdots, B_{m_{1}+\cdots+m_{k+1}}^{(k+1)}\right\}\right. \\
\quad=R_{A_{k+1}^{+} A_{+}^{(k)}} \operatorname{Lop}\left\{A_{+}^{(k+1)} ; B_{1}^{(k+1)}, \cdots, B_{m_{1}+\cdots+m_{k+1}}^{(k+1)}\right\}
\end{gathered}
$$

Therefore

$$
\begin{aligned}
& R_{A_{k+1}^{+} A_{+}^{(k)}} \operatorname{Lop}\left\{A_{1}^{(k+1)} ; B_{1}^{(k+1)}, \cdots, B_{m_{1}+\cdots+m_{k+1}}^{(k+1)}\right\} \\
& =R_{A_{+}^{(k)} A_{A^{+1}}} R_{A_{k^{+}+1} A^{(k)}} \prod_{i<j \leqslant k} R_{A_{t}^{+} A_{j}^{+}} \prod_{\substack{l \neq j \\
l, j<k+1}} R_{A_{i}^{+} A_{j}^{-}} \prod_{l=1}^{k+1} \operatorname{Lop}\left\{A_{l}^{+} ; B_{l 1}, \cdots, B_{l m}\right\} \\
& =R_{A_{k_{1}^{+}+1}^{+} A_{t}^{(k)}} \prod_{i<j>k+1} R_{A_{i}^{+} A_{j}^{+}} \prod_{\substack{l \neq j \\
i, j \leqslant k+1}} R_{A_{i}^{+} A_{j}^{-}} \prod_{l=1}^{k+1} \operatorname{Lop}\left\{A_{l}^{+} ; B_{l 1}, \cdots, B_{l m}\right\} .
\end{aligned}
$$

Lemma 1.2. We assume that $\left\{A_{l}\right\}_{l=1, \cdots, N}$ are hyperbolic polynomials and satisfy the condition iii). Then we have for $u \in \mathcal{E}_{L^{2}}^{2 m+s}\left(R_{+}^{n}\right)$

$$
c_{s}\|u\|_{s+2 m, \tau} \leqslant \sum_{l=1}^{N}\left\|Q_{l}\left(x, y ; \tau, D_{s}, D_{y}\right) u\right\|_{s+2 m_{l}, \tau} \quad \text { for } \operatorname{Im} \tau<0,|\tau|>C_{s},
$$

where $s \geqslant-\min \left\{2 m_{1}, \cdots, 2 m_{N}\right\}$.

Proof. The case whei $N=2$ is showi in Theorem 2.1 in \$1. We have

$$
\begin{aligned}
& Q_{1}^{(k)}\left(x, y ; \tau, D_{x}, D_{y}\right)=A_{k}\left(x, y ; \tau, D_{x}, D_{y}\right) Q_{1}^{(k-1)}\left(x, y ; \tau, D_{\wedge}, D_{y}\right) \\
& \text { + lower order terms, } \\
& Q_{2}^{(k)}\left(x, y ; \tau, D_{1}, D_{y}\right)=A_{k}\left(x, y ; \tau, D_{x}, D_{v}\right) Q_{2}^{(k-1)}\left(x, y ; \tau, D_{1}, D_{y}\right)+\text { l.o.t., } \\
& Q_{t h-1}^{(p)}\left(x, y ; \tau, D_{v}, D_{v}\right)=A_{p}\left(x, y, \tau, D_{v}, D_{v}\right) Q_{k-1}^{(k-1)}\left(x, y ; \tau, D_{\mathrm{r}}, D_{y}\right)+\text { l.o.t., } \\
& Q_{k}^{(k)}\left(x, y ; \tau, D_{x}, D_{y}\right)=A_{1}\left(x, y ; \tau, D_{x}, D_{y}\right) Q_{1}^{(k-1)}\left(x, y ; \tau, D_{y}, D_{y}\right)+\text { l.o.t. } \\
& =A_{2}\left(x, y ; \tau, D_{2}, D_{y}\right) Q_{2}^{(k-1)}\left(x, y ; \tau, D_{x}, D_{y}\right)+\text { l.o.t. }
\end{aligned}
$$




$$
=A_{k-1}\left(x, y ; \tau, D_{x}, D_{y}\right) Q_{k-1}^{(k-1)}\left(x, y ; \tau, D_{x}, D_{y}\right)+\text { 1.o.t. }
$$

Then we have for $1 \leqslant j \leqslant k-1$

$$
\begin{gathered}
\left\|Q_{j}^{(k)} u\right\|_{s+2 m_{m}, \tau}+\left\|\left.Q_{k}^{(k)} u\right|_{\|_{s+2 m_{k}, \tau}}+\right\| u \|_{s+2\left(m_{1}+\cdots+m_{k}\right)-1, \tau} \\
\geqslant c_{s}\left\|Q_{j}^{(k-1)} u\right\|_{s+2 m_{j}+2 m_{k}, \tau},
\end{gathered}
$$

therefore we have

$$
\sum_{j=1}^{k}\left\|Q_{j}^{(k)} u\right\|_{s+2 m_{j}, \tau} \geqslant c_{s}^{\prime} \sum_{j=1}^{k-1}\left\|Q_{j}^{(k-1)} u\right\|_{s+2 m_{j}+2 m_{k}, \tau} .
$$

Here we have only to use the mathematical induction for $k$.

Proposition 1.1. We assume the conditions i), ii), iii) for $\left\{L_{l}\right\}_{l=1, \cdots, N}$. Then $L$ becomes also $\tau$-elliptic $(\operatorname{Im} \tau<0)$ and has the energy inequality of hyperbolic type.

Proof. $\tau$-ellipticity $(\operatorname{Im} \tau<0)$ of $L$ follows Lemma 1.1 .

To show the hyperbolic energy inequality for $L$, we denote

$$
\begin{aligned}
& A\left(x, y ; \tau, D_{x}, D_{y}\right) \\
& \quad=A_{1}\left(x, y ; \tau, D_{x}, D_{y}\right) Q_{1}\left(x, y ; \tau, D_{x}, D_{y}\right)+A_{1}^{\prime}\left(x, y ; \tau, D_{x}, D_{y}\right) \\
& \quad=\cdots \cdots \cdots \cdots \cdots \\
& \quad=A_{N}\left(x, y ; \tau, D_{x}, D_{y}\right) Q_{N}\left(x, y ; \tau, D_{x}, D_{y}\right)+A_{N}^{\prime}\left(x, y ; \tau, D_{x}, D_{y}\right) \\
& B_{m_{1}+\cdots+m_{l-1}+k}\left(x, y ; \tau, D_{x}, D_{y}\right) \\
& \quad=B_{l k}\left(y ; \tau, D_{x}, D_{y}\right) Q_{l}\left(x, y ; \tau, D_{x}, D_{y}\right)+B_{m_{1}+\cdots+m_{l-1}+k}^{\prime}\left(x, y ; \tau, D_{x}, D_{y}\right),
\end{aligned}
$$

where the order of $A^{\prime}$ is less than $2 m$ and the order of $B_{j}^{\prime}$ is less than $r_{j}$. Now we denote

$$
\begin{array}{ll}
A\left(x, y ; \tau, D_{x}, D_{y}\right) u=f, & \\
\left.B_{i}\left(x, y ; \tau, D_{x}, D_{y}\right) u\right|_{x=0}=g_{j} & (j=1, \cdots, m), \\
Q_{l}\left(x, y ; \tau, D_{x}, D_{y}\right) u=u_{l} \quad(l=1, \cdots, N),
\end{array}
$$

then we have

$$
\begin{aligned}
& A_{l} u_{l}=f-A_{l}^{\prime} u, \\
& \left.B_{l k} u_{l}\right|_{x=0}=g_{m_{1}+\cdots+m_{l-1}+k}-\left.B_{m_{1}+\cdots+m_{l-1}+k}^{\prime} u\right|_{x=0} \quad\left(k=1, \cdots, m_{l}\right) .
\end{aligned}
$$

Since each $L_{l}$ has the energy inequality of hyperbolic type, we have 


$$
\begin{aligned}
\left\|u_{l}\right\|_{2 m_{l}-1, \tau} \leqslant & \frac{C}{|\operatorname{Im} \tau|}\left\{\|f\|+\left\|A_{l}^{\prime} u\right\|+\sum_{k=1}^{m}\left\langle g_{m_{1}+\cdots+m_{l-1}+k}\right\rangle_{2 m_{l}-r_{l k}-\frac{1}{2}, \tau}\right. \\
& +\sum_{k=1}^{m_{l}}\left\langle\left. B_{m_{1}+\cdots m_{l-1}+k}^{\prime} u\right|_{x=0}\right\rangle_{2 m_{l}-r_{l k}-\frac{1}{2}}, \\
\leqslant & \frac{C^{\prime}}{|\operatorname{Im} \tau|}\left\{\|f\|+\sum_{j=m_{l}+\cdots+m_{l-1}+1}^{m_{1}+\cdots+m_{l}}\left\langle g_{j}\right\rangle_{2 m-r_{j}-\frac{1}{2}}+\|u\|_{2 m-1, \tau}\right\}
\end{aligned}
$$

for $\operatorname{Im}_{\tau}<-\gamma_{0}(l=1, \cdots, N)$. Summing up them with respect to $l$, we apply Lemma 1.2 .

Example. Let us consider

$$
\begin{aligned}
A_{a}(\tau, \xi, \eta) & =\tau^{2}-2 \tau \xi-\left\{a \xi^{2}+(a+1) \eta^{2}\right\} \quad(a>0) \\
& =\left\{\tau-\left(\xi+\sqrt{(a+1)\left(\xi^{2}+\eta^{2}\right)}\right\}\left\{\tau-\left(\xi-\sqrt{(a+1)\left(\xi^{2}+\eta^{2}\right)}\right)\right\} .\right.
\end{aligned}
$$

The common root of $\left\{A_{a}, A_{a^{\prime}}\right\}\left(a \neq a^{\prime}\right)$ with respect to $\xi$ must satisfy

$$
a^{\prime} A_{a}-a A_{a^{\prime}}=\left(a^{\prime}-a\right)\left(\tau^{2}-2 \tau \xi-\eta^{2}\right)=0,
$$

therefore

$$
\operatorname{Im} \xi=\frac{\eta^{2}+|\tau|^{2}}{2|\tau|^{2}} \operatorname{Im} \tau
$$

that is, no common root is on the upper half plane, whenever $\operatorname{Im} \tau<0$.

Now let

$$
L_{j}=\left\{A_{a_{j}} ; 1\right\} \quad(j=1, \cdots, N),
$$

where $0<a_{1}<a_{2}<\cdots<a_{N}$. Then $\left\{L_{j}\right\}_{j=1, \cdots, N}$ satisfy i), ii), iii).

\section{Hyperbolic energy (continued).}

In the preceding section, we assumed i), ii), iii) on $L$, but (II) of iii) seems too strong restriction on $A$. In this section, we assume i), ii) on $L$. If we try to drop (II) of iii), $A$ is forced to be combined with another appropriate boundary conditions $\left\{C_{j}\right\}_{j=1, \cdots, m}$ of different type from $\left\{B_{j}\right\}_{j=1, \cdots, m}$. Let us find sufficient condition on $\left\{C_{j}\right\}$, in order to get the hyperbolic energy for $M=\left\{A ; C_{1}, \cdots, C_{m}\right\}$, where the degree of $C_{j}$ is $\lambda_{j}$. For the simplicity, we assume that $M(0, y ; \tau, \xi, \eta)$ is independent of $y$ for $|y|>R$.

For each point $(y, \tau, \eta), A_{l}(0, y ; \tau, \xi, \eta)$ is written by

$$
A_{l}(0, y ; \tau, \xi, \eta)=S_{l}(y ; \tau, \xi, \eta) P_{l}(y ; \tau, \xi, \eta)
$$


where $\left\{S_{l}, P_{l}\right\}$ are polynomials of $\xi$, the roots of $S_{l}$ are also the roots of $Q_{l}=\prod_{k \neq l} A_{k}$, and the roots of $P_{l}$ are not the roots of $Q_{l}$. We denote $S=\prod_{l=1}^{N} S_{l}$, the degree of $S_{l}$ is $\mu_{l}$ and the degree of $S$ is $\mu$.

Here we assume

iii) $)^{\prime}(0) \quad M$ is $\tau$-elliptic $(\operatorname{Im} \tau<0)$,

(I) $\inf _{\substack{(x, y) \in R_{n}^{n} \\(\xi, \eta) \in R^{n}}}\left|\prod_{i<j} R_{A_{i} A}^{0}(x, y ; \xi, \eta)\right| \neq 0$,

(II) $\operatorname{Lop}\left\{S_{+}(y ; \tau, \xi, \eta) ; C_{j_{1}}(y ; \tau, \xi, \eta), \cdots, C_{j_{\mu_{+}}}(y ; \tau, \xi, \eta)\right\} \neq 0$

for $y \in R^{n-1}, \operatorname{Im} \tau \leqslant 0, \eta \in R^{n-1}\left(\mu_{+}=\right.$degree of $\left.S_{+}\right)$.

Lemma 2.1. We assume iii)', then we have for $u \in \mathcal{E}_{L^{2}}^{2 m+s}\left(R_{+}^{u}\right)$

$$
\begin{aligned}
c_{s}\|u\|_{s+2 m, \tau} & \leqslant \sum_{l=1}^{N}\left\|Q_{l}\left(x, y ; \tau, D_{x}, D_{y}\right) u\right\|_{s+2 m_{l}, \tau} \\
& +\sum_{j=1}^{m}\left\langle\left. C_{j}\left(y ; \tau, D_{x}, D_{y}\right) u\right|_{x=0}\right\rangle_{s+2 m-\lambda_{j}, \tau}
\end{aligned}
$$

for $\operatorname{Im} \tau<0,|\tau|>C_{s}(s \geqslant 0)$.

Proof. Let us denote

$$
D=\left\{y \in R^{n-1}, \operatorname{Im} \tau \leqslant 0, \eta \in \mathbb{R}^{n-1} ;|y| \leqslant R,|\tau|+|\eta|=1\right\},
$$

and let $\left(y_{0}, \tau_{0}, \eta_{0}\right) \in D$. Since $S_{l}\left(y_{0} ; \tau_{0}, \xi, \eta_{0}\right)$ and $P_{l}\left(y_{0} ; \tau_{0}, \xi, \eta_{0}\right)$ have no common root, there exists a neighbourhood $V$ of $\left(y_{0}, \tau_{0}, \eta_{0}\right)$ in $D$, where $\widetilde{S}_{l}(y ; \tau, \xi, \eta)$ and $\widetilde{P}_{l}(y ; \tau, \xi, \eta)$ are polynomials in $\xi$ with smooth coefficients in $V$, the degrees of them are invariable and

$$
\begin{aligned}
& \widetilde{S}_{l}\left(y_{0} ; \tau_{0}, \xi, \eta_{0}\right)=S_{l}\left(y_{0} ; \tau_{0}, \xi, \eta_{0}\right), \\
& \widetilde{P}_{l}\left(y_{0} ; \tau_{0}, \xi, \eta_{0}\right)=P_{l}\left(y_{0} ; \tau_{0}, \xi, \eta_{0}\right), \\
& A_{l}(y ; \tau, \xi, \eta)=\widetilde{S}_{l}(y ; \tau, \xi, \eta) \widetilde{P}_{l}(y ; \tau, \xi, \eta),
\end{aligned}
$$

and moreover

$$
\begin{aligned}
& \quad \inf _{(y, \tau, \eta) \in V}\left|\prod_{i<j} R_{P_{P} \widetilde{P}_{j}}^{+}(y ; \tau, \eta)\right| \neq 0, \\
& \inf _{(y, \tau, \eta) \in V}\left|\operatorname{Lop}\left\{\widetilde{S}_{+}(y ; \tau, \xi, \eta) ; C_{j_{1}}(y ; \tau, \xi, \eta), \cdots, C_{\mu_{+}}(y ; \tau, \xi, \eta)\right\}\right| \neq 0,
\end{aligned}
$$


where $\widetilde{S}=\prod_{l=-1}^{N} \widetilde{S}_{l}$. Since $D$ is compact, $D$ is covered by finite number of $\left\{V_{j}\right\}$ with the same properties as above.

At first we assume that $A$ is independent of $y$, and that support of $\tilde{u}(\tau, x, \eta)$ is contained in $V$. Since $\widetilde{S}_{l} Q_{l}=\widetilde{S} \widetilde{T}_{l}$, where $\widetilde{T}_{l}=\prod_{k \neq l} \widetilde{P}_{k}$, we have

$$
\begin{gathered}
c_{s} \int_{\imath+j+|\nu|=s+2 m_{l}-\mu_{l}}\left|\tau^{i} \eta^{\nu} D_{x}^{j} \widetilde{T}_{l}\left(\tau, D_{x}, \eta\right) \widetilde{S}\left(\tau, D_{x}, \eta\right) \tilde{u}(\tau, x, \eta)\right|^{2} d x d \eta \\
\leqslant\left\|Q_{l}\left(\tau, D_{x}, D_{y}\right) u(\tau, x, y)\right\|_{s+2 m_{l}, \tau}^{2}
\end{gathered}
$$

therefore we have from Lemma 1.2

$$
\begin{array}{r}
c_{s}^{\prime} \int_{i+j+|\nu|=s+2 m-\mu}\left|\tau^{i} \eta^{\nu} D_{x}^{j} \widetilde{S}\left(\tau, D_{x}, \eta\right) \tilde{u}(\tau, x, \eta)\right|^{2} d x d \eta \\
\leqslant \sum_{l=1}^{N}\left\|Q_{l}\left(\tau, D_{x}, D_{y}\right) u(\tau, x, y)\right\|_{s+2 m l, \tau}^{2} .
\end{array}
$$

Then we have from Proposition 3.1 in $\$ 1$

$$
c_{s}^{\prime \prime}\|u\|_{s+2 m, \tau} \leqslant \sum_{l=1}^{N}\left\|Q_{l}\left(\tau, D_{x}, D_{y}\right) u\right\|_{s+2 n l, \tau}+\sum_{k=1}^{\mu_{+}}\left\langle\left. C_{j_{k}}\left(\tau, D_{x}, D_{y}\right) u\right|_{x=0}\right\rangle_{s+2 m-\lambda_{j_{k}}, \tau} .
$$

In the general case, we use two kinds of the partition of unity for $R_{+}^{n}$ and for $\left\{\operatorname{Im} \tau \leqslant 0, \eta \in R^{n-1} ;|\tau|+|\eta|=1\right\}$. We refer to appendix about the treatment of the partition of unity in $(\tau, \eta)$-space.

Proposition 2. 1 . We assume ii) and iii)' on $M$, and moreover $\left(\begin{array}{c}B_{1}(y ; \tau, \xi, \eta) \\ \vdots \\ B_{m}(y ; \tau, \xi, \eta)\end{array}\right)=\left(\begin{array}{c}c_{11}(y ; \tau, \eta) \cdots c_{1 n}(y ; \tau, \eta) \\ \cdots \ldots \ldots \cdots \cdots \cdots \cdots \\ c_{m 1}(y ; \tau, \eta) \cdots c_{m n}(y ; \tau, \eta)\end{array}\right)\left(\begin{array}{c}c_{1}(y ; \tau, \xi, \eta) \\ \vdots \\ c_{m}(y ; \tau, \xi, \eta)\end{array}\right)$

where $c_{i j}(y ; \tau, \eta)$ are homogeneous polynomials of $(\tau, \eta)$ with coefficienis in $\mathscr{B}\left(\mathbb{R}^{n-1}\right)$. Then $M$ has hyperbolic energy ineauality.

Proof. It is similar to the proof of Proposition 1.1, only we remark that

$$
\sum_{j=1}^{n}\left\langle\left. B_{j}\left(y ; \tau, D_{1}, D_{y}\right) u\right|_{x=0}\right\rangle_{2 m-,-\frac{1}{1}, \tau} \leqslant C \sum_{j=1}^{m}\left\langle\left. C_{j}\left(y ; \tau, D_{x}, D_{y}\right) u\right|_{x-0}\right\rangle_{2 m-\lambda,-\frac{1}{2}, \tau}
$$

and we use Lornma 2.1 instead of Lemma 1.1.

Example. Let us consider

$$
A_{a}(\tau, \xi, \eta)=\tau^{2}-a x(\xi, \eta) \quad(a>0),
$$


where $\alpha(\xi, \eta)=\xi^{2}+h(\eta) \xi+k(\eta)$ is positive definite. The common root of $\left\{A_{a}, A_{a^{\prime}}\right\}\left(a \neq a^{\prime}\right)$ only appears on $\tau=0$.

Now let

$$
\begin{aligned}
& L_{j}=\left\{A_{a_{j}} ; 1\right\} \quad j=1, \cdots, N \quad\left(0<a_{1}<\cdots<a_{N}\right), \\
& A=\prod_{j=1}^{N} A_{a_{j}}, \quad B_{j}=\prod_{l \neq j} A_{a_{l}} \quad(j=1, \cdots, N), \quad C_{j}=\alpha^{j-1} \quad(j=1, \cdots, N), \\
& M=\left\{A ; C_{1}, \cdots, C_{N}\right\},
\end{aligned}
$$

then

$$
\left(\begin{array}{c}
B_{1} \\
\vdots \\
B_{N}
\end{array}\right)=\left(\begin{array}{c}
\tau^{2 N}-\sum_{j=2}^{N} a_{j} \tau^{2(N-1)} \cdots(-1)^{N} \prod_{j=2}^{N} a_{i} \\
\cdots \cdots \cdots \cdots \cdots \cdots \cdots \cdots \cdots \cdots \cdots \cdots \cdots \cdots \cdots \cdots \cdots \\
\tau^{2 N}-\sum_{j=1}^{N-1} a_{j} \tau^{2(N-1)} \cdots(-1)^{N-1} \prod_{j=1}^{N-1} a_{j}
\end{array}\right)\left(\begin{array}{c}
1 \\
\vdots \\
\alpha^{N-1}
\end{array}\right),
$$

and

$$
\left\{\begin{array}{lll}
S_{l}=1, & P_{l}=A_{a_{l}} & \text { for } \tau \neq 0, \\
S_{l}=A_{a_{l}}, & P_{l}=1 & \text { for } \tau=0 .
\end{array}\right.
$$

Let $A_{+}(\xi)=\prod_{j=1}^{N}\left(\xi-\xi_{j}\right)$, then from Lemma 3.1 in $\xi 1$,

$$
\operatorname{Lop}\left\{A_{+} ; 1, \alpha, \cdots, \alpha^{N-1}\right\}=\prod_{i>j} \frac{\alpha\left(\xi_{i}\right)-\alpha\left(\xi_{j}\right)}{\xi_{i}-\xi_{j}}=\prod_{i>j}\left\{\left(\xi_{i}+\xi_{j}\right)+h(\eta)\right\} \neq 0 .
$$

If $\operatorname{Im} \xi_{j}>0(j=1, \cdots, N)$. Then $M$ satisfies ii $)$ and iii $)^{\prime}$.

\section{Existence theorem.}

We say that $L u=F$ is analytically solvable in the region $\operatorname{Im} \tau<-r$, if $L u=F$ is not only solvable in $\operatorname{Im} \tau<-\gamma$, but $u$ is analytic in $\operatorname{Im} \tau<-r$ with values $\mathcal{E}_{L 2}^{2 m-1}\left(R_{+}^{n}\right)$, whenever $F$ is analytic in $\operatorname{Im} \tau<-\gamma$ with values in $\mathfrak{S}_{0}$.

Proposition 3.1. We assume that

i) $L$ is $\tau$-elliptic $(\operatorname{Im} \tau<0)$,

ii) $L$ has the energy inequality of hyperbolic type in $\operatorname{Im} \tau<-\gamma$. Then $L u=F$ is analytically solvable in $\operatorname{Im} \tau<-\gamma$.

Proof. From i), applying Theorem 4.2 in $\S 1, L$ is solvable in $\operatorname{Im} \tau<0$ and $|\tau|>C_{\text {arg } \tau}$. Now we fix a solvable point $\tau_{0}\left(\operatorname{Im} \tau_{0}<-\gamma\right)$, 
and make Taylor's expansion on $L$ and $F$ at $\tau_{0}$. Denoting $\tau=\tau_{0}+\mu$, we have

$$
\begin{aligned}
& L=L_{0}+\mu L_{1}+\cdots+\mu^{2 m} L_{2 m}, \\
& F=F_{0}+\mu F_{1}+\mu^{2} F_{2}+\cdots \quad \text { for } \quad|\mu|<\rho_{0}\left(=-\operatorname{Im} \tau_{0}-\gamma\right) .
\end{aligned}
$$

Then $L_{0} u=F_{0}$ is solvable and

$$
\left\|L_{k} u\right\|_{0, \tau_{0}} \leqslant C\|u\|_{2 m-1, \tau_{0}} \quad(k=1,2, \cdots, 2 m),
$$

where $C$ is independent of $\tau_{0}$.

Now we solve the problems:

$$
\begin{aligned}
L_{0} u_{0} & =F_{0}, \\
L_{0} u_{1} & =F_{1}-L_{1} u_{0}, \\
\quad \cdots \cdots \cdots \cdots & \cdots \cdots \\
L_{0} u_{k} & =F_{k}-\left(L_{1} u_{k-1}+\cdots+L_{2 m} u_{k-2 m}\right),
\end{aligned}
$$

We have from ii)

$$
\begin{gathered}
\left\|u_{k}\right\|_{2 m-1, \tau_{0}} \leqslant \frac{C}{\left|\operatorname{Im} \tau_{0}\right|}\left\{\left\|F_{k}\right\|_{0, \tau_{0}}+\left\|L_{1} u_{k-1}\right\|_{0, \tau_{0}}+\cdots+\left\|L_{2 m} u_{k-2 m}\right\|_{0, \tau_{0}}\right\} \\
\leqslant C^{\prime}\left\{\left\|F_{k}\right\|_{0, \tau_{0}}+\left\|u_{k-1}\right\|_{2 m-1, \tau_{0}}+\cdots+\left\|u_{k-2 m}\right\|_{2 m-1, \tau_{0}}\right\},
\end{gathered}
$$

where $C, C^{\prime}$ are independent of $\tau_{0}$. Then

$$
\begin{gathered}
\sum_{k=0}^{l}|\mu|^{k}\left\|u_{k}\right\|_{2 m-1, \tau_{0}} \leqslant C^{\prime}\left\{\sum_{k=0}^{l}|\mu|^{k}\left\|F_{k}\right\|_{0, \tau_{0}}+(|\mu|\right. \\
\left.\left.+\cdots+|\mu|^{2 m}\right) \sum_{k=0}^{l-1}|\mu|^{k}\left\|u_{k}\right\|_{2 m-1, \tau_{0}}\right\} .
\end{gathered}
$$

Let $\rho_{1}<\min \left(1, \frac{1}{2 m C^{\prime}}, \rho_{0}\right)$, then

$$
\sum_{k=0}^{\infty}|\mu|^{k}\left\|u_{k}\right\|_{2 m-1, \tau_{0}} \leqslant C\left(\rho_{1}\right) \sum_{k=0}^{\infty}|\mu|^{k}\left\|F_{k}\right\|_{0, \tau_{0}} \text { for }|\mu|<\rho_{1} .
$$

Then $\sum_{k=0}^{l} \mu^{k} u_{k}$ converges to $u$ in $\mathcal{E}_{L 2}^{2 m-1}\left(R_{+}^{n}\right)$ for $|\mu|<\rho_{1}$.

On the other hand

$$
\begin{gathered}
L \sum_{k=0}^{l} \mu^{k} u_{k}=F_{0}+\mu F_{1}+\cdots+\mu^{l} F_{l}+\mu^{l+1}\left(L_{1} u_{l}+L_{2} u_{l-1}+\cdots+L_{2 m} u_{l-2 m+1}\right) \\
+\mu^{l+2}\left(L_{2} u_{l}+\cdots+L_{2 m} u_{l-2 m+2}\right)+\cdots+\mu^{l+2 m} L_{2 m} u_{l},
\end{gathered}
$$

then 


$$
\left\|L \sum_{k=0}^{l} \mu^{k} u_{k}-\sum_{k=0}^{l} \mu \mu^{k} F_{k}\right\|_{0, r_{0}} \leqslant 2 m C \sum_{k-l-2 m+1}^{l}|\mu|^{k}\left\|u_{k}\right\|_{2 m-1, \tau} \underset{l \rightarrow \infty}{\longrightarrow} 0
$$

for $|\mu| \leqslant \rho_{1}$. From i), applying Theorem 4.1 in $\$ 1$, $\imath$ belongs to $\mathcal{E}_{L 2}^{2 m}\left(R_{+}^{n}\right)$.

Finally we remark that $\rho_{1}$ deperds orly on $\rho_{0}$, relating to $\tau_{0}$. So in this way, $u$ can be extended analytically on the region $\operatorname{Im} \tau<-\gamma$.

\section{Appendix}

1. Singular integral operators witp positive parameter $\lambda$.

At first we consider $H(\lambda, \xi)$, which is homogenecus of degree zero with respect to $(\lambda, \xi) \in R_{+}^{1} \times \mathbb{R}^{n}$, and we denote

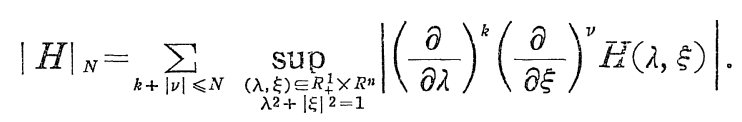

Let us dencte

$$
\begin{aligned}
& \hat{u}(\xi)=\mathscr{F}[u(x)]=\int_{R^{n}} e^{-t x \xi} u(x) d x, \\
& u(x)=\mathscr{F}^{-1}[\hat{u}(\xi)]=\frac{1}{(2 \pi)^{n}} \int_{R^{a}} e^{i x \xi} \hat{u}(\xi) d \xi,
\end{aligned}
$$

for $u \in S\left(R^{n}\right)$ or more generally for $u \in S^{\prime}\left(R^{n}\right)$. Here we define

$$
\begin{aligned}
H(\lambda, D) u(x) & =\mathscr{F}^{-1}[H(\lambda, \xi) \hat{u}(\xi)], \\
A^{s}(\lambda, D) u(x) & =\mathscr{F}^{-1}\left[\{\Lambda(\lambda, \xi)\}^{s} \hat{u}(\xi)\right]=\mathscr{F}^{-1}\left[\left(\sqrt{\lambda^{2}+|\xi|^{2}}\right)^{s} \hat{u}(\xi)\right] \\
\text { for } & u \in \mathcal{S}\left(R^{n}\right) \quad \text { (s: complex number). }
\end{aligned}
$$

Now we denote

$$
H_{s}(\lambda, x)=\mathscr{F}^{-1}\left[H(\lambda, \xi)\{\Lambda(\lambda, \xi)\}^{s}\right],
$$

then we have

Lemma 1.1. Let $r>0$,

$$
\left|H_{s}(\lambda, x)\right| \leqslant C|H|_{n+\left[l_{0}+\gamma\right]_{+1}}|x|^{-(n+\operatorname{Re} s)}(\lambda|x|)^{-\left(\gamma+l_{0}-\operatorname{Re} s\right)}
$$

for $\operatorname{Re} s \leqslant l_{0}$, where $C=C\left(n, l_{0}, r\right)$ is independent of $s$.

Proof. Since we have 


$$
x^{\nu} H_{s}(\lambda, x)=\mathscr{F}^{-1}\left[\left(i \frac{\partial}{\partial \xi}\right)^{\nu}\left\{H(\lambda, \xi) \Lambda(\lambda, \xi)^{s}\right\}\right]
$$

and

$$
\begin{aligned}
& \left|\left(i \frac{\partial}{\partial \xi}\right)^{\nu}\left\{H(\lambda, \xi) \Lambda(\lambda, \xi)^{s}\right\}\right| \leqslant C(n,|\nu|)|H|_{|\nu|}(\lambda+|\xi|)^{\mathrm{Rcs}-|\nu|}, \\
& \left|e^{i x \xi}-1\right| \leqslant 2|x \xi|^{\theta} \quad(0 \leqslant \theta \leqslant 1),
\end{aligned}
$$

we have for $|\nu|>n+\operatorname{Re} s$

$$
\begin{aligned}
\left|x^{\nu} H_{s}(\lambda, x)\right| & =\left|\frac{1}{(2 \pi)^{n}} \int\left(e^{i \lambda \xi}-1\right)\left(i \frac{\partial}{\partial \xi}\right)^{\nu}\left\{H(\lambda, \xi) \Lambda(\lambda, \xi)^{s}\right\} d \xi\right| \\
& \leqslant C\left(n, l_{0}, \gamma\right)|H|_{n+\left[l_{0}+\gamma\right] \mid 1}|x|^{1-\left(l_{0}+\gamma-\left[l_{0}+\gamma\right]\right)} \lambda^{\text {Res }-l_{0}-\gamma} .
\end{aligned}
$$

Now we denote the norm of $a(x) \in \mathscr{B}^{N+\alpha}\left(R^{n}\right)$ by

$$
|a|_{N+\alpha}=\sum_{|\nu| \leqslant N} \sup _{x \in R^{n}}\left|\left(\frac{\partial}{\partial x}\right)^{\nu} a(x)\right|+\sum_{|\nu|=N} \sup _{a, y \in R^{n}} \frac{\left|\left(\frac{\partial}{\partial x}\right)^{\nu} a(x)-\left(\frac{\partial}{\partial y}\right)^{\nu} a(y)\right|}{|x-y|^{\alpha}} \text {. }
$$

Lemma 1.2. Let $N$ be a non-negative integer, $0 \leqslant \alpha<\beta<1$, $a(x) \in \mathscr{B}^{N+\beta}\left(R^{n}\right)$. Then we have for $u \in \mathcal{S}\left(\mathbb{R}^{n}\right)$

$$
\begin{aligned}
& H(\lambda, D) \Lambda^{N+\alpha}(\lambda, D) a(x) u(x) \\
& =\sum_{|\nu| \leqslant N} \frac{N !}{(N-|\nu|) ! \nu !} a^{(\nu)}(x) \mathbb{R}^{\nu}(\lambda, D) H(\lambda, D) \Lambda^{N+\alpha-|\nu|}(\lambda, D) u(x) \\
& \quad+\sum_{\left|\nu_{0}\right|+|\nu| \leqslant N} \int G_{\nu_{0} \nu}(\lambda, x, y) \lambda^{\nu 0} D^{\nu} u(y) d y,
\end{aligned}
$$

where

$$
a^{(\nu)}(x)=D^{\nu} a(x), \quad K^{\nu}(\lambda, \xi)=\left(\frac{\xi}{\Lambda(\lambda, \xi)}\right)^{\nu} .
$$

Moreover, there exist positive consiants $C, \varepsilon$, such that

$$
\left|G_{\nu_{0} \nu}(\lambda, x, y)\right| \leqslant C|a|_{N+\beta}|H|_{\mu+1} K_{\varepsilon}(\lambda, x-y) \quad \text { for } \lambda \geqslant 1,
$$

where

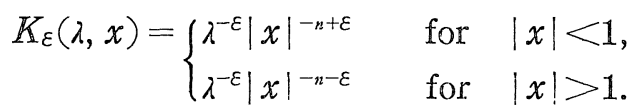

Proof. At first we consider $H \Lambda^{N+s}$ for $\operatorname{Re} s<0$. Since

$$
\begin{aligned}
\Lambda(\lambda, \xi) & =\frac{\lambda}{\Lambda(\lambda, \xi)} \lambda+\frac{\xi_{1}}{\Lambda(\lambda, \xi)} \xi_{1}+\cdots+\frac{\xi_{n}}{\Lambda(\lambda, \xi)} \xi_{n} \\
& =R_{0}(\lambda, \xi) \lambda+R_{1}(\lambda, \xi) \xi_{1}+\cdots+R_{n}(\lambda, \xi) \xi_{n},
\end{aligned}
$$


we have

$$
\begin{aligned}
& H(\lambda, D) \Lambda^{N+s}(\lambda, D) a(x) u(x) \\
& =H(\lambda, D) \Lambda^{s}(\lambda, D) \sum_{\nu_{0}+|\nu|+N} \frac{N !}{\nu_{0} ! \nu !}\left\{R_{0}(\lambda, D) \lambda\right\}^{\nu_{0}}\{R(\lambda, D)\}^{\nu} \\
& \quad \times \sum_{\mu+\kappa=\nu} \frac{\nu !}{\mu ! \kappa !} D^{\mu} a(x) D^{\kappa} u(x) \\
& =\sum_{\nu_{0}+|\mu|+|\kappa|=N} \frac{N !}{\nu_{0} ! \mu ! \kappa !} \int\left(H R_{0}^{\nu_{0}} R^{\mu+\kappa}\right)_{s}(\lambda, x-y) a^{(\mu)}(y) \lambda^{\nu_{0}} D^{\kappa} u(y) d y \\
& =\sum_{|\mu| \leqslant N} a^{(\mu)}(x) \frac{N !}{(N-|\mu|) ! \mu !} H R^{\mu} \Lambda^{N+s-|\mu|} u(x) \\
& \quad+\sum_{\nu_{0}+|\kappa| \leqslant N} \int G_{\nu_{0 \kappa}}^{s}(\lambda, x, y) \lambda^{\nu_{0}} D^{\kappa} u(y) d y .
\end{aligned}
$$

From Lemma 1.1, we have

$$
\begin{aligned}
& \left|G_{\nu_{0} \kappa}^{s}(\lambda, x, y)\right| \\
& =\left|\sum_{|\mu|=N-\nu_{0}-|\kappa|} \frac{N !}{\nu_{0} ! \mu ! \kappa !}\left(H R_{0}^{\nu_{0}} R^{\mu+\kappa}\right)_{s}(\lambda, x-y)\left\{a^{(\mu)}(y)-a^{(\mu)}(x)\right\}\right| \\
& \quad \leqslant C|H|_{n+[\alpha+\gamma]+1}|a|_{N+\beta} \lambda^{-(\gamma+\alpha-\operatorname{Re} s)}|x-y|^{-n-\gamma-\alpha+\beta} .
\end{aligned}
$$

Then we have for $\operatorname{Re} s \leqslant \alpha$ and $\lambda \geqslant 1$, taking $\gamma=\gamma_{0}\left(0<\gamma_{0}<\beta-\alpha\right)$,

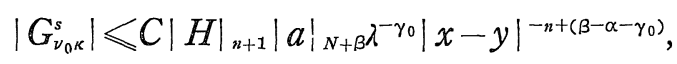

taking $\gamma=\gamma_{1}\left(\beta-\alpha<\gamma_{1}<1-\alpha\right)$,

$$
\left|G_{\nu_{0} \kappa}^{s}\right| \leqslant C|H|_{n+1}|a|_{N+\beta} \lambda^{-\gamma_{1}}|x-y|^{-n-\left(\gamma_{1}-\beta+\alpha\right)} \text {. }
$$

Now we put $\varepsilon=\min \left(\gamma_{0}, \beta-\alpha-\gamma_{0}, \gamma_{1}-\beta+\alpha\right)$, then we have

$$
\left|G_{\nu_{0} \kappa}^{s}\right| \leqslant C|H|_{n+1}|a|_{N+\beta} K_{\varepsilon}(x-y) \text { for } \operatorname{Re} s \leqslant \alpha .
$$

From this estimate, we can use the method of analytic continuation for $\operatorname{Re} s \leqslant \alpha$, in the representation formula.

Remark. We define

$$
K_{\varepsilon, N}(u)=\sum_{\nu_{0}+|\nu| \leqslant N} \int K_{\varepsilon}(\lambda, x-y)\left|\lambda^{\nu_{0}} D^{\nu} u(y)\right| d y,
$$

then

$$
\left\|K_{\varepsilon, N}(u)\right\| \leqslant C \lambda^{-\varepsilon}\left\|\Lambda^{N}(\lambda, D) u\right\|
$$


Corollary. Let $0<3 \varepsilon<1-\alpha$, then we have for $u \in \mathcal{S}\left(R^{n}\right)$

$$
\begin{aligned}
& \left\|H(\lambda, D) \Lambda^{N+\alpha}(\lambda, D) a(x) u(x)-a(x) H(\lambda, D) \Lambda^{N+\alpha}(\lambda, D) u(x)\right\| \\
& \quad \leqslant C_{\varepsilon} \lambda^{-\varepsilon}|a|_{N+\alpha+2 \varepsilon}|H|_{n+1}\left\|\Lambda^{N}(\lambda, D) u\right\| .
\end{aligned}
$$

Let $H(x ; \lambda, \xi)$ be homogeneous of degree 0 with respect to $(\lambda, \xi) \in R_{+}^{1} \times R^{n}$, and denote

$$
\begin{aligned}
& |H|_{\alpha, k}=|H|_{\mathcal{B}^{\alpha, k}\left(R^{n}, S_{+}^{n}\right)}=\sum_{\substack{|\mu| \leqslant[\alpha] \\
l+|\nu| \leqslant k}} \sup _{\substack{x \in R^{n} \\
(\lambda, \xi) \in S_{+}^{n}}}\left|\left(\frac{\partial}{\partial x}\right)^{\mu}\left(\frac{\partial}{\partial \lambda}\right)^{l}\left(\frac{\partial}{\partial \xi}\right)^{\nu} H(x ; \lambda, \xi)\right| \\
& +\sum_{\substack{|\mu|=[\alpha] \\
l+|\nu| \leqslant k}} \sup _{\substack{x, y \in R^{n} \\
(\lambda, \xi) \in S_{+}^{n}}} \frac{\left|\left(\frac{\partial}{\partial x}\right)^{\mu}\left(\frac{\partial}{\partial \lambda}\right)^{l}\left(\frac{\partial}{\partial \xi}\right)^{\nu} H(x ; \lambda, \xi)-\left(\frac{\partial}{\partial y}\right)^{\mu}\left(\frac{\partial}{\partial \lambda}\right)^{l}\left(\frac{\partial}{\partial \xi}\right)^{\nu} H(y ; \lambda, \xi)\right|}{|x-y|^{\alpha-[\alpha]}} .
\end{aligned}
$$

Then there exists an extension $\widetilde{H}(x ; \lambda, \xi)$ of $H(x ; \lambda, \xi)$ which is homogeneous of degree 0 with respect to $(\lambda, \xi) \in R^{1} \times R^{n}$, satisfying

$$
|\widetilde{H}|_{\alpha, k}=|\widetilde{H}|_{\mathcal{B}^{\alpha, k}\left(R^{n}, S^{n}\right)} \leqslant C|H|_{\alpha, k} .
$$

Now we denote spherical harmonics on $S^{n}$ of order $m$ by $Y_{m k}(\lambda, \xi)$ $\left(k=1,2, \cdots, k_{m}\right)$, and briefly denote $Y_{m k}=\varphi_{k_{0}+k_{1}+\cdots+k_{m-1}+k}$. Then $\left\{\varphi_{j}\right\}_{j=1,2, \cdots}$ is a complete orthonormal system in $L^{2}\left(S^{n}\right)$.

Here we denote

$$
a_{j}(x)=\int_{S^{n}} \widetilde{H}(x ; \lambda, \xi) \varphi_{j}(\lambda, \xi) d S,
$$

then we have

Lemma 1.3. For $\alpha, k \geqslant 0$,

$$
\left.\left.\sum_{j=1}^{\infty}\left|a_{j}\right|_{\alpha}\right|_{\varphi_{j}}\right|_{k} \leqslant C|\widetilde{H}|_{\alpha, B(k)},
$$

where $\beta(k)=2\left(\left[\frac{3 n+2 k}{4}\right]+1\right)$.

Proof. We use the well known properties about $\left\{\varphi_{j}\right\}$ :

$$
\begin{aligned}
& \left|a_{j}\right|_{\alpha} \leqslant C|H|_{\alpha, 2 N} \lambda_{j}^{-N} \quad(N=0,1,2, \cdots), \\
& \left|\varphi_{j}\right|_{k} \leqslant C \lambda_{j}^{\frac{1}{2}\left(k+\frac{1}{2} n+\varepsilon\right)} \quad(\varepsilon>0) \text {, } \\
& \sum_{j=1}^{\infty} \lambda_{j}^{-\frac{1}{2} n-\varepsilon}<+\infty \quad(\varepsilon>0) \text {. }
\end{aligned}
$$


Therefore we have

$$
\left.\left.\left|a_{j}\right|_{\alpha}\right|_{\varphi_{j}}\right|_{k} \leqslant C|\widetilde{H}|_{\alpha, 2 N} \lambda_{j}^{-N+\frac{1}{2}\left(k+\frac{1}{2} n+\varepsilon\right)},
$$

whose sum converges for $N>\frac{2 k+3 n}{4}$.

Now remarking Lemma 1.3 and

$$
\left\|a_{j}(x) \frac{1}{(2 \pi)^{n}} \int e^{i x \xi} \varphi_{j}(\lambda, \xi) \hat{u}(\xi) d \xi\right\| \leqslant C\left|a_{j}\right|_{0}\left|\varphi_{j}\right|_{0}\|u\|,
$$

we define for $\widetilde{H} \in \mathscr{B}^{0, B(0)}\left(R^{n}, S^{n}\right)$,

$$
\widetilde{H}(x ; \lambda, D) u(x)=\sum_{j=1}^{\infty} a_{j}(x) \frac{1}{(2 \pi)^{n}} \int e^{i x \xi} \varphi_{j}(\lambda, \xi) \hat{u}(\xi) d \xi \quad \text { for } u \in \mathcal{S}\left(R^{n}\right)
$$

then we have

$$
\|\widetilde{H}(x ; \lambda, D) u\| \leqslant C|\widetilde{H}|_{0, B(0)}\|u\|
$$

Since $\sum_{j=1}^{N} a_{j}(x) \varphi_{j}(\lambda, \xi)$ converges to $\widetilde{H}(x ; \lambda, \xi)$ in $\mathscr{B}^{0,0}\left(R^{n}, S^{n}\right)$ as $N \rightarrow \infty$,

$$
\sum_{j=1}^{N} a_{j}(x) \frac{1}{(2 \pi)^{n}} \int e^{i x \xi} \varphi_{j}(\lambda, \xi) \hat{u}(\xi) d \xi
$$

converges to

$$
\frac{1}{(2 \pi)^{n}} \int e^{i x \xi} \widetilde{H}(x ; \lambda, \xi) \hat{u}(\xi) d \xi
$$

in $\mathscr{B}^{0}\left(R^{n}\right)$ as $N \rightarrow \infty$, which implies that $\widetilde{H}(x ; \lambda, D)=0$ for $\lambda>0$, if $\widetilde{H}(x ; \lambda, \xi)=0$ for $\lambda>0$. Hence we define for $H \in \mathscr{B}^{0, B(0)}\left(R^{n}, S_{+}^{n}\right)$

$$
H(x ; \lambda, D) u(x)=\widetilde{H}(x ; \lambda, D) u(x) \quad(\lambda>0) \text { for } u \in \mathcal{S}\left(R^{n}\right),
$$

which is independent of extensions. We say that $H(x ; \lambda, D)$ is a singular integral operator with symbol $H(x ; \lambda, \xi)$.

Lemma 1.4.

i) Let $l_{1} \geqslant 0$, then there exist $C>0, \varepsilon>0$, such that

$$
\begin{aligned}
& \| H_{1}(x ; \lambda, D) \Lambda^{l_{1}}(\lambda, D) H_{2}(x ; \lambda, D) \Lambda^{l_{2}}(\lambda, D) u(x) \\
& \quad-\left(H_{1} \circ H_{2}\right)(x ; \lambda, D) \Lambda^{1_{1}+l_{2}} u(x) \| \\
& \quad \leqslant C \lambda^{-\varepsilon}\left|H_{1}\right|_{0, \beta(n+1)}\left|H_{2}\right|_{l_{1}+2 \varepsilon, \beta(0)}\left\|\Lambda^{\left[l_{1}\right]^{+l_{2}}} u\right\| \text { for } \lambda \geqslant 1,
\end{aligned}
$$

where $\left(H_{1} \circ H_{2}\right)(x ; \lambda, D)$ is a singular integral operator with symbol $H_{1}(x ; \lambda, \xi) H_{2}(x ; \lambda, \xi)$. 
ii) $\left\|H(x ; \lambda, D)^{*} u(x)-H^{\ddagger}(x ; \lambda, D) u(x)\right\| \leqslant C \lambda^{-\varepsilon}|H|_{2 \varepsilon, \beta(n+1)}\|u\|$ for $\lambda \geqslant 1$, where $H^{*}$ implies the adjoint of $H$ in $L^{2}\left(R^{n}\right)$, 黑and $H^{*}(x ; \lambda, D)$ is a

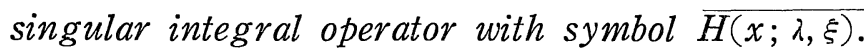

Proof. i) $H_{1} \Lambda^{t_{1}} H_{2} \Lambda^{t_{2}} u$

$$
=\sum_{j} a_{1 j}(x) \varphi_{j}(\lambda, D) \Lambda^{l_{1}}(\lambda, D) \sum_{k} a_{2 k}(x) \varphi_{k}(\lambda, D) \Lambda^{l_{2}}(\lambda, D) u .
$$

From Lemma 1.2, we have

$$
\begin{aligned}
& \left\|a_{1 j} \varphi_{j} \Lambda^{l_{1}} a_{2 k} \varphi_{k} \Lambda^{l_{2}} u-a_{1 j} a_{2 k} \varphi_{j} \Lambda^{l_{1}} \varphi_{k} \Lambda^{l_{2}} u\right\| \\
& \quad \leqslant C \lambda^{-\varepsilon}\left|a_{1 j}\right|_{0}\left|a_{2 k}\right|_{l_{1+2} \varepsilon}\left|\varphi_{j}\right|_{n+1}\left|\varphi_{k}\right|_{0}|| \Lambda^{\left[l_{1}\right]^{+l_{2}}} u \| .
\end{aligned}
$$

Then we have only to apply Lemma 1.3.

ii) $H(x ; \lambda, D)^{*} u(x)=\sum_{j} \varphi_{j}(\lambda, D) \overline{a_{j}(x)} u(x)$.

From Lemma 1.2, we have

$$
\left\|\varphi_{j} \bar{a}_{j} u-\bar{a}_{j} \varphi_{j} u\right\| \leqslant C \lambda^{-\varepsilon}\left|a_{j}\right|_{2 \varepsilon}\left|\varphi_{j}\right|_{n+1}\|u\| .
$$

Next we consider $H(x ; \lambda, D)$, relating to decomposition of unity.

Lemma 1.5. Let $l \geqslant 0$ and $\beta(x)=1$ on the support of $\alpha(x)$. Then

$$
\begin{aligned}
& H(x ; \lambda, D) A^{l}(\lambda, D) \alpha(x) u(x) \\
& =\sum_{|\nu| \leqslant[l]} \frac{[l] !}{([l]-|\nu|) ! \nu !} \alpha^{(\nu)}(x) H(x ; \lambda, D) R^{\nu}(\lambda, D) A^{l-|\nu|}(\lambda, D) u(x) \\
& \quad+\beta(x)(T u)(x)+T^{\prime}(\beta u)(x),
\end{aligned}
$$

where

$$
|T u(x)|,\left|T^{\prime} u(x)\right| \leqslant C|\alpha|_{l+2 \varepsilon}|\beta|_{l+2 \varepsilon}|H|_{0, \beta(n+1)} K_{\varepsilon,[l]}(u)(x) .
$$

Proof. $H \Lambda^{l} \alpha u=\sum_{j} a_{j} \varphi_{j} \Lambda^{l} \alpha u$.

From Lemma 1.2, we have

where

$$
\begin{aligned}
a_{j} \varphi_{j} \Lambda^{l} \alpha u & =a_{j} \sum_{|\nu| \leqslant[l]} C_{l \nu} a^{(\nu)} R^{\nu} \varphi_{j} \Lambda^{l-|\nu|} u \\
& +a_{j} \sum_{\nu_{0}+|\nu| \leqslant[l]} \int G_{\nu_{0} \nu}^{\left(\rho_{j} l^{\prime}, \alpha\right)}(\lambda, x, y) \lambda^{\nu} D^{\nu} u(y) d y,
\end{aligned}
$$

$$
\left|G_{\nu_{0 \nu}}^{\left(\varphi_{j} \Lambda^{l}, \alpha\right)}(\lambda, x, y)\right| \leqslant\left.\left. C|\alpha|_{l_{+2} \varepsilon}\right|_{\varphi_{j}}\right|_{n+1} K_{\varepsilon}(\lambda, x-y) .
$$

Now we denote

$$
G_{\nu_{0} \nu}^{\left(H \Lambda^{\prime}, \alpha\right)}(\lambda, x, y)=\sum_{j=1}^{\infty} a_{j}(x) G_{\nu \nu}^{\left(1, \Lambda^{l}, \alpha\right)}(\lambda, x, y),
$$


then

where

$$
\begin{aligned}
H \Lambda^{l} \alpha u & =\sum_{|\nu| \leqslant[l]} C_{l \nu} \alpha^{(\nu)}(x) H(x ; \lambda, D) R^{\nu}(\lambda, D) \Lambda^{l-|\nu|} u(x) \\
& +\sum_{\nu_{0}+|\nu| \leqslant[l]} \int G_{\nu_{0} \nu}^{(H A l, \alpha)}(\lambda, x, y) \lambda^{\nu_{0}} D^{\nu} u(y) d y,
\end{aligned}
$$

$$
\left|G_{\nu_{0} \nu}^{(H A l, \alpha)}(\lambda, x, y)\right| \leqslant C|\alpha|_{l+2 \varepsilon}|H|_{0, B(n+1)} K_{\varepsilon}(\lambda, x-y) .
$$

Now

$$
\begin{aligned}
H \Lambda^{l} \alpha u= & H \Lambda^{l} \alpha \beta u=\sum_{|\nu| \leqslant[l]} C_{l \nu} \alpha^{(\nu)}(x) H(x ; \lambda, D) R^{\nu}(\lambda, D) \Lambda^{l-|\nu|} \beta(x) u(x) \\
& +\sum_{\nu_{0}+|\nu| \leqslant[l]} \int G_{\nu_{0} \nu}^{\left(H \lambda^{l}, \alpha\right)}(\lambda, x, y) \lambda^{\nu_{0}} D^{\nu}(\beta(y) u(y)) d y \\
= & \sum_{|\nu| \leqslant l l]} C_{l \nu} \alpha^{(\nu)}(x) H(x ; \lambda, D) R^{\nu}(\lambda, D) \Lambda^{l-|\nu|} u(x) \\
& +\sum_{|\nu| \leqslant[l]} C_{l \nu} \alpha^{(\nu)}(x) \sum_{\mu_{0}+|\mu| \leqslant[l]-|\nu|} \int G_{\mu_{0} \mu}^{\left(H R^{\nu} A^{l-\mid \nu l}, \beta\right)}(\lambda, x, y) \lambda^{\mu_{0}} D^{\mu} u(y) d y \\
& +\sum_{\nu_{0}+|\nu| \leqslant[l]} \int G_{\nu_{0} \nu}^{\left(H A^{l}, \alpha\right)}(\lambda, x, y) \lambda^{\nu_{0}} D^{\nu}(\beta(y) u(y)) d y \\
= & \sum_{|\nu| \leqslant[l]} C_{l \nu} \alpha^{(\nu)} H R^{\nu} \Lambda^{l-|\nu|} u \\
& +\beta(x) \sum_{\mu_{0}+|\mu| \leqslant[l]} \int G_{\mu_{0} \mu}(\lambda, x, y) \lambda^{\mu_{0}} D^{\mu} u(y) d y \\
& +\sum_{\nu_{0}+|\nu| \leqslant[l]} \int G_{\nu_{0} \nu}^{\prime}(\lambda, x, y) \lambda^{\nu_{0}} D^{\nu}(\beta(y) u(y)) d y,
\end{aligned}
$$

where

$$
\begin{aligned}
& \left|G_{\mu_{0} \mu}(\lambda, x, y)\right|=\left|\sum_{|\nu| \leqslant[l]-\mu_{0}-|\mu|} C_{l \nu} \alpha^{(\nu)}(x) G_{\mu_{0} \mu}^{\left(H R^{\nu A^{l}-|\nu|}, \beta\right)}(\lambda, x-y)\right| \\
& \leqslant C|\alpha|_{[\ell]}|\beta|_{l+2 \varepsilon \mid}|H|_{0, \beta(n+1) K \varepsilon}(\lambda, x-y), \\
& \left|G_{\nu_{\nu}}^{\prime}(\lambda, x, y)\right|=\left|G_{\nu_{0} \nu}^{(H A l, \alpha)}(\lambda, x, y)\right| \leqslant C|\alpha|_{l+2 \varepsilon}|H|_{0, \beta(n+1)} K_{\varepsilon}(\lambda, x-y) \text {. }
\end{aligned}
$$

Let $\left\{\alpha_{k}(x)\right\}_{k=1,2, \ldots}$ be a decomposition of unity in $R^{n}$, such that

$$
\begin{aligned}
& \sum_{k=1}^{\infty} \alpha_{k}(x)^{2}=1, \quad \alpha_{k}(x)=\alpha\left(x-x_{k}\right), \\
& \alpha(x) \geqslant 0, \quad \alpha(x)=0 \text { for }|x|>\delta, \quad|\alpha|_{l+2 \varepsilon} \leqslant K(0<3 \varepsilon<[l]+1-l) .
\end{aligned}
$$

Lemma 1. 6. Let $l \geqslant 0, r>0$, then

$$
\begin{gathered}
\sum_{k=1}^{\infty}\left\|H\left(x_{k} ; \lambda, D\right) \Lambda^{l}(\lambda, D) \alpha_{k}(x) u(x)-\alpha_{k}(x) H(x ; \lambda, D) \Lambda^{l}(\lambda, D) u(x)\right\|^{2} \\
\leqslant C|H|_{\gamma, \beta(n+1)}^{2}\left(\lambda^{-\varepsilon}\left\|\Lambda^{[l]} u\right\|+\delta^{\gamma}\left\|\Lambda^{l} u\right\|\right)^{2} \quad \text { for } \lambda \geqslant 1,
\end{gathered}
$$

where $C=C(K, \varepsilon)$. 
Proof. Relating to $\left\{\alpha_{k}(x)\right\}$ we consider $\left\{\beta_{k}\right\},\left\{r_{k}\right\}$, suct that

$$
\begin{array}{ll}
\beta(x) \alpha(x)=\alpha(x), & \gamma(x) \beta(x)=\beta(x), \\
\beta(x) \geqslant 0, & \gamma(x) \geqslant 0, \\
|\beta|_{1+2 \varepsilon} \leqslant K^{\prime}, & |\gamma|_{l^{2} 2 \varepsilon \leqslant K^{\prime},} \\
\beta_{k}(x)=\beta\left(x-x_{k}\right), & \gamma_{k}(x)=\gamma\left(x-x_{k}\right), \\
\sum_{k=1}^{\infty} \beta_{k}\left(x^{2}\right) \leqslant K^{\prime \prime}, & \sum_{k=1}^{\infty} \gamma_{k}(x)^{2} \leqslant K^{\prime \prime} .
\end{array}
$$

Now we denote

$$
\begin{aligned}
& H\left(x_{k} ; \lambda, D\right) \Lambda^{l}(\lambda, D) \alpha_{k}(x) u(x)-\alpha_{k}(x) H(x ; \lambda, D) \Lambda^{l}(\lambda, D) u(x) \\
&=\left\{H\left(x_{k} ; \lambda, D\right) \Lambda^{l}(\lambda, D) \alpha_{k}(x)-\alpha_{k}(x) H\left(x_{k} ; \lambda, D\right) \Lambda^{l}(\lambda, D)\right\} \beta_{k}(x) u(x) \\
&+\alpha_{k}(x)\left\{H\left(x_{k} ; \lambda, D\right)-H(x ; \lambda, D)\right\} \Lambda^{l}(\lambda, D) \beta_{k}(x) u(x) \\
&+\alpha_{k}(x)\left\{H(x ; \lambda, D) \Lambda^{l}(\lambda, D) \beta_{k}(x)-\beta_{k}(x) H(x ; \lambda, D) \Lambda^{l}(\lambda, D)\right\} u(x) \\
&= I_{k 1}+I_{k 2}+I_{k 3} .
\end{aligned}
$$

We have

$$
\begin{aligned}
& \left\|I_{k 1}\right\| \leqslant C|H|_{0, \beta(n+1)} \lambda^{-\varepsilon}\left\|\Lambda^{[l]}\left(\beta_{k} u\right)\right\| \quad \text { (from Lemma 1.4), } \\
& \left\|I_{k 2}\right\| \leqslant C\left|\alpha_{k}(x) H\left(x_{k} ; \lambda, \xi\right)-\alpha_{k}(x) H(x ; \lambda, \xi)\right|_{0, \beta(0)} \\
& \quad \times\left\|\Lambda^{l} \beta_{k} u\right\| \leqslant C \delta^{\gamma}|H|_{\gamma, \beta(0)}\left\|\Lambda^{l}\left(\beta_{k} u\right)\right\|, \\
& \left\|I_{k 3}\right\| \leqslant C\left\{\sum_{1=|\nu|<[l]}\left\|\gamma_{k} H R^{\nu} \Lambda^{l-|\nu|} u\right\|+|H|_{0, \beta(n+1)}\left(\left\|\gamma_{k} K_{\varepsilon,[l]}(u)\right\|\right.\right. \\
& + \\
& \left.\left.+K_{\varepsilon,[l]}\left(\gamma_{k} u\right) \|\right)\right\} \quad \text { (from Lemma 1.5). }
\end{aligned}
$$

Now we have

$$
\begin{aligned}
& \sum_{k=1}^{\infty}\left\|r_{k} K_{\varepsilon,[l]}(u)\right\|^{2} \leqslant C\left\|K_{\varepsilon,[l]}(u)\right\|^{2} \leqslant C \lambda^{-2 \varepsilon}\left\|\Lambda^{[l]} u\right\|^{2}, \\
& \sum_{k=1}^{\infty}\left\|K_{\varepsilon,[l]}\left(\gamma_{k} u\right)\right\|^{2} \leqslant C \sum_{k=1}^{\infty} \lambda^{-2 \varepsilon}\left\|\Lambda^{[l]}\left(\gamma_{k} u\right)\right\|^{2} \leqslant C \lambda^{-2 \varepsilon}\left\|\Lambda^{[l]} u\right\|^{2} .
\end{aligned}
$$

Moreover, since

$$
\left\|\Lambda^{\prime} \beta_{k} u\right\|^{2} \leqslant C\left\{\sum_{|\nu| \leqslant[l]}\left\|\gamma_{k}^{(\nu)} R^{v} \Lambda^{l-|\nu|} u\right\|^{2}+\left\|\gamma_{k} K_{\varepsilon,[l]}(u)\right\|^{2}+\left\|K_{\varepsilon,[l]}\left(\gamma_{k} u\right)\right\|^{2}\right\},
$$

we have

$$
\sum_{k=1}^{\infty}\left\|\Lambda^{l} \beta_{k} u\right\|^{2} \leqslant C\left\|\Lambda^{l} u\right\|^{2}
$$

Here we have

$$
\sum_{k=1}^{\infty}\left(\left\|I_{k 1}\right\|+\left\|I_{k 2}\right\|+\left\|I_{k 3}\right\|\right)^{2} \leqslant C|H|_{\gamma, \beta(n+1)}^{2}\left(\lambda^{-\varepsilon}\left\|\Lambda^{[l]} u\right\|^{1}+\delta^{\gamma}\left\|\Lambda^{l} u\right\|\right)^{2} .
$$




\section{2. $\lambda$-elliptic $(\lambda<0)$ operator in $\boldsymbol{R}_{+}^{n}$ with singular integral boundary conditions.}

Let us consider

$$
L(x, y ; \lambda, \xi, \eta)=\left\{A(x, y ; \lambda, \xi, \eta) ; B_{1}(y ; \lambda, \xi, \eta), \cdots, B_{m}(y ; \lambda, \xi, \eta)\right\},
$$

where

$$
\begin{gathered}
A(x, y ; \lambda, \xi, \eta)=\sum_{i+j+|\nu|=2, n} a_{i j \nu}(x, y) \lambda^{i} \xi^{j} \eta^{\nu}\left(a_{i j \nu}(x, y) \in \mathscr{B}\left(R_{+}^{n}\right)\right), \\
=\xi^{2 m}+a_{1}(x, y ; \lambda, \eta) \Lambda(\lambda, \eta)+\cdots+a_{2 m}(x, y ; \lambda, \eta) \Lambda(\lambda, \eta)^{2 m}, \\
B_{j}(y ; \lambda, \xi, \eta)=\sum_{k=1}^{2 m} b_{j k}(y ; \lambda, \eta) \Lambda^{r,-(2 m-k)}(\lambda, \eta) \xi^{2 m-k} \\
\left(b_{j k}(y ; \lambda, \eta) \in \mathscr{B}\left(R^{n-1} \times S_{+}^{n-1}\right)\right),
\end{gathered}
$$

and $L(x, y ; \lambda, \xi, \eta)$ is $\lambda$-elliptic $(\lambda>0)$ in $R_{+}^{n}$ in the sense stated in $\S 4$ of $\S 1$. Now we define

$$
\begin{aligned}
& A\left(x, y ; \lambda, D_{x}, D_{y}\right)=\sum_{t+j+|\nu|=2 m} a_{i j \nu}(x, y) \lambda^{\prime} D_{x}^{i} D_{y}^{\nu}, \\
& B_{j}\left(y ; \lambda, D_{x}, D_{y}\right)=\sum_{k=1}^{2 m} b_{j k}\left(y ; \lambda, D_{y}\right) \Lambda^{r,-(2 m-k)}\left(\lambda, D_{y}\right) D_{x}^{2 m-k}, \\
& \quad L\left(x, y ; \lambda, D_{x}, D_{y}\right) \\
& \quad=\left\{A\left(x, y ; \lambda, D_{x}, D_{y}\right) ; B_{1}\left(y ; \lambda, D_{x}, D_{y}\right), \cdots, B_{m}\left(y ; \lambda, D_{x}, D_{y}\right)\right\} .
\end{aligned}
$$

Lemma 2.1. Let

$$
B\left(y ; \lambda, D_{x}, D_{y}\right)=b\left(y ; \lambda, D_{y}\right) \Lambda^{r+\frac{1}{2}}\left(\lambda, D_{y}\right) D_{x}^{s},
$$

where $b$ is a singular integral operator in $R^{n-1}$ and $r, s$ are nonnegaive integer, then we have

$$
\begin{gathered}
\sum_{k=1}^{\infty}\left\langle B\left(y_{k} ; \lambda, D_{x}, D_{y}\right) \alpha_{k}(x, y) u(x, y)-\alpha_{k}(x, y) B\left(y ; \lambda, D_{x}, D_{y}\right) u(x, y)\right\rangle^{2} \\
\leqslant C|b|_{\gamma, \beta(n)}^{2}\left(\lambda^{-\varepsilon}+\delta^{\gamma}\right)^{2}\|u\|_{r+s+1, \lambda}^{2},
\end{gathered}
$$

where $\left\{\alpha_{k}\right\}$ is a partition of unity in $R^{n}$ stated in Section 1.

Proof.

$$
\begin{gathered}
B\left(y_{k} ; \lambda, D_{x}, D_{y}\right) \alpha_{k}(x, y) u(x, y)-\alpha_{k}(x, y) B\left(y ; \lambda, D_{x}, D_{y}\right) u(x, y) \\
=b\left(y_{k} ; \lambda, D_{y}\right) A^{r-\frac{1}{2}}\left(\lambda, D_{y}\right) \sum_{t=0}^{s} \frac{s !}{(s-t) ! t !}\left(D_{x}^{s-t} \alpha_{k}(x, y)\right) D_{x}^{t} u(x, y) \\
\quad-\alpha_{k}(x, y) b\left(y ; \lambda, D_{y}\right) \Lambda^{r+\frac{1}{2}}\left(\lambda, D_{y}\right) D_{x}^{s} u(x, y)
\end{gathered}
$$




$$
\begin{aligned}
=\sum_{t=0}^{s} & \frac{s !}{(s-t) ! t !}\left\{b\left(y_{k} ; \lambda, D_{y}\right) A^{r+\frac{1}{2}}\left(\lambda, D_{y}\right) \alpha_{k}^{(s-t)}(x, y) D_{x}^{t} u(x, y)\right. \\
& \left.-\alpha_{k}^{(s-t)}(x, y) b\left(y ; \lambda, D_{y}\right) A^{r+\frac{1}{2}}\left(\lambda, D_{y}\right) D_{x}^{t} u(x, y)\right\} \\
& +\sum_{t=0}^{s-1} \frac{s !}{(s-t) ! t !} \alpha_{k}^{(s-t)}(x, y) b\left(y ; \lambda, D_{y}\right) \Lambda^{r+\frac{1}{2}}\left(\lambda, D_{y}\right) D_{\lambda}^{t} u(x, y) .
\end{aligned}
$$

Here we apply Lemma 1.6.

\section{Corollary.}

$$
\begin{aligned}
& \sum_{k=1}^{\infty} \| L\left(x_{k}, y_{k} ; \lambda, D_{x}, D_{y}\right) \alpha_{k}(x, y) u(x, y) \\
& \quad-\alpha_{k}(x, y) L\left(x, y ; \lambda, D_{x}, D_{y}\right) u(x, y) \|_{s, \lambda}^{2} \\
& \leqslant C_{s}\left(\lambda^{-\varepsilon}+\delta^{y}\right)^{2}\|u\|_{2 m+s, \lambda}^{2} \quad \text { for } \lambda \geqslant 1 \quad(s \text { : non-negative integer }) .
\end{aligned}
$$

Here we have

Proposition 2.1. Let $L$ be $\lambda$-elliptic $(\lambda>0)$ in $R_{+}^{n}$, then

$$
\|u\|_{2 m+s, i} \leqslant C_{s}\left(\|L u\|_{s, \lambda}+\|u\|\right) \quad \text { for } \lambda>0,
$$

where $u \in \mathcal{E}_{L 2}^{2 m+s}\left(R_{+}^{n}\right)$ ( $s$ : non-negative integer).

Next we define the adjoint system of $L$. At first we define

$$
\begin{aligned}
& B_{j}(y ; \lambda, \xi, \eta)=\xi^{r-m} A_{+}(0, y ; \lambda, \xi, \eta)=\sum_{k=1}^{2 m} b_{j \xi}(y ; \lambda, \eta) A^{r,-(2 m-k)}(\lambda, \eta) \xi^{2 m-k}, \\
& B_{j}\left(y ; \lambda, D_{\imath}, D_{y}\right)=\sum_{k-1}^{2 m} b_{j k}\left(y ; \lambda, D_{y}\right) \Lambda^{r,-(2 m-k)}\left(\lambda, D_{y}\right) D_{x}^{2 m-k} \\
& \left(r_{j}=j-1, j=m+1, m+2, \cdots, 2 m\right),
\end{aligned}
$$

and together with $\left\{B_{j}\right\}_{j=1,2, \cdots, m}$,

$$
\left(\begin{array}{c}
\Lambda^{2 m-1-r_{1}} B_{1}\left(y ; \lambda, D_{x}, D_{y}\right) \\
\Lambda^{2 m-1-r_{2}} B_{2}\left(y ; \lambda, D_{x}, D_{y}\right) \\
\vdots \\
\Lambda^{2 m-1-r_{2} m} B_{2 m}\left(y ; \lambda, D_{x}, D_{y}\right)
\end{array}\right)=\mathscr{B}\left(\begin{array}{c}
D_{\lambda}^{2 m-1} \\
\Lambda\left(\lambda, D_{y}\right) D_{x}^{2 m-2} \\
\vdots \\
\Lambda^{2 m-1}\left(\lambda, D_{y}\right)
\end{array}\right),
$$

where

$$
\mathscr{B}=\left(\Lambda^{2 m-1-1},\left(\lambda, D_{y}\right) b_{j k}\left(y ; \lambda, D_{y}\right) \Lambda^{-\left(2 m-1-r_{\jmath}\right)}\left(\lambda, D_{y}\right)\right)_{j, k=1,2, \cdots, 2 m} .
$$

Let

$$
\mathscr{B}=\mathscr{B}_{0}+\mathscr{B}_{1}
$$

where

$$
\mathscr{B}_{0}=\left(b_{j k}\left(y ; \lambda, D_{y}\right)\right)_{j, k=1, \cdots, 2 m},
$$




$$
\left\langle\mathscr{B}_{1} U\right\rangle_{s, \lambda} \leqslant C \lambda^{-\varepsilon}\langle U\rangle_{s, \lambda} \quad(\lambda \geqslant 1) .
$$

Lemma 2. 2. There exists $\mathscr{H}$ for $\lambda>\lambda_{0}$, such that

$$
\left(\begin{array}{r}
D_{x}^{2 m-1} \\
\Lambda\left(\lambda, D_{y}\right) D_{x}^{2 m-2} \\
\vdots \\
\Lambda^{2 m-1}\left(\lambda, D_{y}\right)
\end{array}\right)=\mathscr{H}\left(\begin{array}{c}
\Lambda^{2 m-1-r_{1}}\left(\lambda, D_{y}\right) B_{1}\left(y ; \lambda, D_{x}, D_{y}\right) \\
\Lambda^{2 m-1-r_{2}}\left(\lambda, D_{y}\right) B_{2}\left(y ; \lambda, D_{x}, D_{y}\right) \\
\vdots \\
\Lambda^{2 m-1-r_{2} m}\left(\lambda, D_{y}\right) B_{2 m}\left(y ; \lambda, D_{x}, D_{y}\right)
\end{array}\right),
$$

where

$$
\begin{aligned}
& \mathscr{H}=\mathscr{H}_{0}+\mathscr{H}_{1}, \\
& \mathscr{H}_{0}(y ; \lambda, \eta)=\mathscr{B}_{0}(y ; \lambda, \eta)^{-1}, \\
& \left\langle\mathscr{H}_{1} U\right\rangle_{s, \lambda} \leqslant C \lambda^{-\varepsilon}\langle U\rangle_{s, \lambda} .
\end{aligned}
$$

Proof. Let

$$
\mathscr{H}_{0}\left(y ; \lambda, D_{y}\right) \mathscr{B}_{0}\left(y ; \lambda, D_{y}\right)-I=\mathscr{R}_{1},
$$

then we have from Lemma 1.4

$$
\left\langle\mathscr{R}_{1} U\right\rangle_{s, \lambda} \leqslant C \lambda^{-\varepsilon}\langle U\rangle_{s, \lambda} \quad(\lambda \geqslant 1) .
$$

Here we have

$$
\mathcal{H}_{0} \mathscr{B}-I=\mathscr{H}_{0} \mathscr{B}_{1}+\mathscr{R}_{1},
$$

where there exists $\left(I+\mathscr{H}_{0} \mathscr{B}_{1}+\mathscr{R}_{1}\right)^{-1}$ for $\lambda>\lambda_{0}$, and then we have

$$
\left(I+\mathscr{H}_{0} \mathscr{B}_{1}+\mathscr{R}_{1}\right)^{-1} \mathscr{H}_{0} \mathscr{B}=I \quad \text { for } \lambda>\lambda_{0} .
$$

Let

$$
\begin{aligned}
\mathscr{H} & =\left(I+\mathscr{H}_{0} \mathscr{B}_{1}+\mathscr{R}_{1}\right)^{-1} \mathscr{H}_{0}=\mathscr{H}_{0}-\left(\mathscr{H}_{0} \mathscr{B}_{1}+\mathscr{R}_{1}\right)\left(I+\mathscr{H}_{0} \mathscr{B}_{1}+\mathscr{R}_{1}\right)^{-1} \mathscr{H}_{0} \\
& =\mathscr{H}_{0}+\mathscr{H}_{1} .
\end{aligned}
$$

Let

$$
\begin{aligned}
A^{(*)}= & D_{x}^{2 m}+D_{x}^{2 m-1} \Lambda\left(\lambda, D_{y}\right) a_{1}\left(x, y ; \lambda, D_{y}\right)^{*}+\cdots \\
& \cdots+\Lambda^{2 m}\left(\lambda, D_{y}\right) a_{2 m}\left(x, y ; \lambda, D_{y}\right)^{*},
\end{aligned}
$$

and $(),(\operatorname{resp}\langle\rangle$,$) be the inner product in L^{2}\left(R_{+}^{n}\right)\left(\operatorname{resp} L^{2}\left(R^{n-1}\right)\right)$.

Lemma 2. 3. Let $u, v \in \mathcal{E}_{L^{2}}^{2 n}\left(R_{+}^{n}\right)$. Then

$$
\begin{aligned}
& (A u, v)-\left(u, A^{(*)} v\right) \\
& \left.\quad=i<\mathcal{A}\left(\begin{array}{c}
D_{x}^{2 m-1} \\
\Lambda\left(\lambda, D_{y}\right) D_{x}^{2 m-2} \\
\vdots \\
\Lambda^{2 m-1}\left(\lambda, D_{y}\right)
\end{array}\right) u,\left(\begin{array}{c}
\Lambda^{-(2 m-1)}\left(\lambda, D_{y}\right) D_{x}^{2 m-1} \\
\Lambda^{-(2 m-2)}\left(\lambda, D_{y}\right) D_{x}^{2 m-2} \\
\vdots \\
1
\end{array}\right) v\right\rangle_{x=0},
\end{aligned}
$$


where

$$
\begin{aligned}
& \mathscr{A}=\mathscr{A}_{0}\left(y ; \lambda, D_{y}\right)+\mathscr{A}_{1},
\end{aligned}
$$

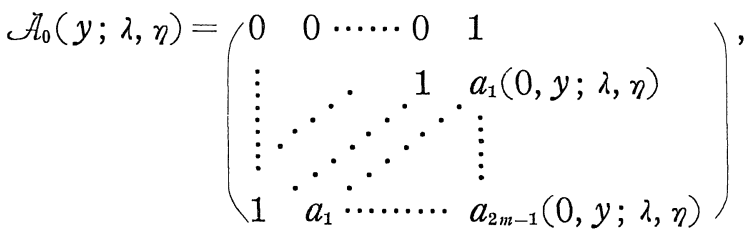

$$
\begin{aligned}
& \left\langle\mathscr{A}_{1} U\right\rangle_{s, \lambda} \leqslant C \lambda^{-\varepsilon}\langle U\rangle_{s, \lambda} \text {. }
\end{aligned}
$$

Proof.

$$
\begin{aligned}
& (A u, v)-\left(u, A^{(*)} v\right)=i \sum_{k=0}^{2 m-1} \sum_{l=0}^{2 m-k-1}\left\langle D_{x}^{2 m-k-1-l} u, D_{x}^{l} \Lambda^{k} a_{k}^{*} v\right\rangle_{x=0} \\
& \quad=i \sum_{k=0}^{2 m-1} \sum_{l=0}^{2 m-k-1} \sum_{h=0}^{l}\left\langle D_{x}^{2 m-k-1-l} u,\left(\begin{array}{l}
l l \\
l
\end{array}\right) \Lambda^{k}\left(D_{x}^{l-h} a_{k}\right) * D_{x}^{h} v\right\rangle_{x=0} \\
& =i \sum_{s=0}^{2 m-1} \sum_{h=0}^{s}\left\langle\Lambda^{h}\left\{\sum_{k=0}^{s-h}\left(\begin{array}{c}
s-k \\
h
\end{array}\right)\left(D_{x}^{s-h-k} a_{k}\right) \Lambda^{-(s-h-k)}\right\} \Lambda^{-h} \Lambda^{s} D_{x}^{2 m-1-s} u, \Lambda^{-h} D_{x}^{h} v\right\rangle_{x=0} \\
& =i \sum_{s=0}^{2 m-1} \sum_{t=s}^{2 m-1}\left\langle\alpha_{s t} \Lambda^{s} D_{x}^{2 m-1-s} u, \Lambda^{-(2 n-1-t)} D_{x}^{2 m-1-t} v\right\rangle_{x-0}
\end{aligned}
$$

where we denote

$$
\mathcal{A}=\left(\alpha_{s t}\right)_{s, t=0,1, \cdots, 2 m-1} .
$$

Now we denote for $\lambda>\lambda_{0}$

$$
\begin{aligned}
& \left(\begin{array}{c}
B_{1}^{\prime} \\
\vdots \\
\dot{B}_{2 m}^{\prime}
\end{array}\right)=\left(\begin{array}{cc}
\Lambda^{2 m-1-r_{1}} & \\
\ddots & \\
& \Lambda^{2 m-1-r_{2 m}}
\end{array}\right)(\mathcal{A} \mathcal{H})^{*}\left(\begin{array}{c}
\Lambda^{-(2 m-1)} D_{x}^{2 m-1} \\
\vdots \\
1
\end{array}\right), \\
& L^{\prime}=\left\{A^{(*)} ; B_{m+1}^{\prime}, \cdots, B_{2 m}^{\prime}\right\}
\end{aligned}
$$

and

$$
\left\|L^{\prime} u\right\|_{s, \lambda}^{\prime}=\left\|A^{(*)} u\right\|_{s, \lambda}+\sum_{j=m+1}^{2 m}\left\langle B_{j}^{\prime} u\right\rangle_{2 m-r_{j}-\frac{1}{2}+s, \lambda},
$$

then we have

Proposition 2. 2. It holds for $\lambda>\lambda_{0}$ that

$$
(A u, v)-\left(u, A^{(*)} v\right)=i \sum_{j=1}^{2 m}\left\langle B_{j} u, B_{j}^{\prime} v\right\rangle_{x=0}
$$

for $u, v \in \mathcal{E}_{L 2}^{2 m}\left(R_{+}^{n}\right)$, and

$$
\|u\|_{2 m+s, \lambda} \leqslant C\left\|L^{\prime} u\right\|_{s, \lambda}^{\prime}
$$

for $u \in \mathcal{E}_{L 2}^{2 m+s}\left(R_{+}^{n}\right)$ (s: non-negative integer). 
Proof. Let

$$
\begin{aligned}
A_{0}^{(*)}(x, y ; \lambda, \xi, \eta) & =\overline{A(x, y ; \lambda, \xi, \eta)}, \\
\left(\begin{array}{c}
B_{10}^{\prime}(y ; \lambda, \xi, \eta) \\
\vdots \\
B_{2 m 0}^{\prime}(y ; \lambda, \xi, \eta)
\end{array}\right)= & \left(\begin{array}{cc}
\Lambda^{2 m-1-r_{1}}(\lambda, \eta) \\
\ddots & \Lambda^{2 m-1-r_{2 m}}(\lambda, \eta)
\end{array}\right)\left(\mathcal{A}_{0} \mathcal{H}_{0}\right) *(y ; \lambda, \eta) \\
& \times\left(\begin{array}{c}
\Lambda^{-(2 m-1)}(\lambda, \eta) \xi^{2 m-1} \\
\vdots \\
1
\end{array}\right),
\end{aligned}
$$

and

$$
L_{0}^{\prime}=\left\{A_{0}^{(*)} ; B_{m+10}^{\prime}, \cdots, B_{2 m 0}^{\prime}\right\} .
$$

Then we have from Proposition 3.2 in $\$ 1$ that $L_{0}^{\prime}$ is $\lambda$-elliptic $(\lambda>0)$. Therefore we can apply Proposition 2.1 on $L_{0}^{\prime}$.

Weak existence theorem for $L$ follows Proposition 2.2, and Proposition 2.1 implies that weak solution of $L$ becomes strong solution, hence we have Theorem 4.2 in $\$ 1$.

\section{References}

[1] Agmon, S., Problèmes mixtes pour les équations hyperboliques d'ordre supérieur, Colloques sur les équations aux dérivées partielles, C.N.R.S. (1962), 13-18.

[2] Mizohata, S. Quelques problèmes au bord, du type mixte, pour des équations hyperboliques, Séminaire subventionne par le C.N.R.S. (R.C.P. 126), Collège de France 1966-1967, 23-60.

[3] Miyatake, S., On the mixed problems for fourth order hyperbolic equations, Proc. Japan Acad. 44 (1968), 257-262.

[4] Schechter, M., Integral inequalities for partial differential operators and functions satisfying general boundary conditions, Comm. Pure Appl. Math. $\mathbb{1 2}$ (1959), 3766. 